\title{
General Study of the Modified Einstein Method of Computing Total Sediment Discharge
}

GEOLOGICAL SURVEY WATER-SUPPLY PAPER 2066 


\section{General Study of the Modified Einstein Method of Computing Total Sediment Discharge}

By D. E. BURKHAM and D. R. DAWDY

GEOLOGICAL SURVEY WATER-SUPPLY PAPER 2066

Method of computing sediment discharge on stream sites

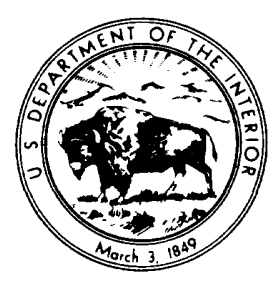




\section{UNITED STATES DEPARTMENT OF THE INTERIOR}

CECIL D. ANDRUS, Secretary

\section{GEOLOGICAL SURVEY}

H. William Menard, Director

\section{Library of Congress Cataloging in Publication Data}

Burkham, D E 1927-

General study of the modified Einstein method of computing total sediment discharge.

(Geological Survey water-supply paper ; 2066)

Bibliography: p. 43-44.

1. Sediment transport. 2. Hydrology--Mathematics. I. Dawdy, David R., 1926- joint author. II. Title. III. Title: Einstein method of computing total Sediment discharge. IV. Series: United States. Geological Survey. Water-supply paper ; 2066.
GB850.B87
$551.3^{\prime} 03$
$80-607076$

For sale by the Superintendent of Documents, U. S. Government Printing Office Washington, D. C. 20402 


\section{CONTENTS}

Conversion factors

Symbols _.

Abstract -...

Introduction -.........- 2

Basis for revision ........ 4

Einstein method

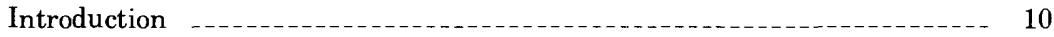

Equations pertinent to suspended-sediment discharge _............... 10

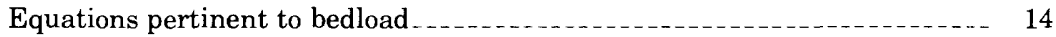

Equations for transition between bedload and suspended load _-...... 15

Modified Einstein procedure .....- 16

Introduction -

Equations for ranges of large particles _............. 16

Equations for ranges of small particles _-_. 17

Revised modified Einstein procedure -

Shear velocity

Bedload _............. 21

Exponent $z$ and total load ............. 22

Comparison of computed and measured sediment discharges _........... 23

Basic data _... 23

Niobrara data

Middle Loup River data ......... 24

Fivemile Creek data

Rio Grande reach _._. 25

Computation procedure

Results of comparison _... 32

Introduction _... 32

Niobrara River ....... 32

Middle Loup River _....... 33

Fivemile Creek at Shoshoni _............ 34

Fivemile Creek at Riverton _......... 34

Rio Grande conveyance channel _........ 35

Characteristics for the five data groups ............... 38

Summary and conclusions ........ 38

References cited

\section{ILLUSTRATIONS}

FiguREs 1-4. Graphs showing:

1. Relation between percentage of bed material finer than size used to represent roughness height, and standard error of estimate for the different resistance equations for flow in rigid-boundary streams 
Figure

2. Relation of roughness parameter to relative smoothness based on 84-percentile size of intermediate diameter of streambed particles

3. Relation between percentage of bed material finer than size used to represent roughness height and the coefficient $C$ in the Keulegan equation

4. Correction $x$ in equation 12 in terms of $k_{s} / \delta$

5. Diagram showing computation of total sediment discharge ...... 6-10. Graphs showing:

6. Vertical distribution of streamflow

7. Approximate relation of $z$ to the ratio of suspendedsediment discharge in the sampling zone to bedload discharge -.............

8. Approximate standard error of estimate for the different bed forms and particle-size ranges, Rio Grande conveyance channel near Bernardo, N. Mex

9. Weighted approximate standard error of estimate for the different ranges of particle sizes and for the total sediment discharge for five data groups

10. Relation between the average of ratios of computed to measured total sediment discharge to the averages of median diameters for five data groups

Page

\section{TABLE}

TABLE 1. Comparison of computed sediment discharge from the modified Einstein procedsre and revised modified Einstein procedure applied to normal sections with measured sediment discharge at contracted section or weir

\section{CONVERSION FACTORS}

For readers who may prefer to use International System (SI) of units, rather than inch-pound units, the conversion factors for the terms used in this report are listed below:

\begin{tabular}{lcl}
\multicolumn{1}{l}{ Multiply inch-pound unit } & $B y$ & To obtain SI (metric) unit \\
& & \\
$\mathrm{ft}$ (foot) & 0.3048 & $\mathrm{~m}$ (meter) \\
$\mathrm{ft} / \mathrm{s}$ (foot per second) & 0.3048 & $\mathrm{~m} / \mathrm{s}$ (meter per second) \\
$\begin{array}{l}\mathrm{ft}^{2} / \mathrm{s} \text { (square foot } \\
\quad \text { per second) }\end{array}$ & 0.0929 & $\mathrm{~m}^{2} / \mathrm{s}$ (square meter \\
$\begin{array}{l}\text { lb/s (pounds per second) } \\
\text { ton/d (ton per day) }\end{array}$ & 456.6 & per second) \\
\end{tabular}




\section{SYMBOLS}

Single prime mark on $A, J$, or $Q$ designates association with the sampling depth

Double prime mark on $A, J, I$, or $Q$ designates association with the total depth through which suspended sediment is discharged

C

$C_{a}$

$C_{S}$

$C_{y}$

$C_{0}$

$C_{1}$

Conc

$d$

$d_{n}$

$d_{s}$

$D$

$D_{16}$

$D_{35}$

$D_{50}$

$D_{50 G}$

$D_{65}$

$D_{84}$

$D_{95}$

e

$f$

g

$i_{b}$

$i_{B}$

$i_{s}$

$i_{\mathrm{T}}$

$i_{s} q_{s}$

$i_{B} q_{B}$

$i_{T} q_{T}$

$i_{B} Q_{B}$

Subscript $M$ designates a parameter associated with the modified Einstein procedure

Subscript $R M$ designates a parameter associated with the revised modified Einstein procedure

Thickness of bed layer

Ratio of bed layer thickness to water depth

Ratio of $d_{n}$ to $d_{s}$

Ratio of $2 D$ to $d$

Constant, scale of $\phi_{*}$

Parameter related to turbulence; equals $1 / \kappa$

Constant, scale of $\psi_{*}$

Constant

Sediment concentration for a particle having a diameter, $D$, at a distance $a$ from the streambed

A variable coefficient which is a function of the median diameter of the bed material

Sediment concentration for a particle having a diameter, $D$, at a distance $y$ from the streambed

A constant

A constant

Measured suspended-sediment concentration

Mean depth of water

Vertical distance not sampled

Vertical distance sampled

Diameter of a particle

Particle size at which 16 percent of the bed material by weight is finer

Particle size at which 35 percent of the bed material by weight is finer

Particle size at which 50 percent of the bed material by weight is finer

A parameter used to represent $D_{50}$; equals $\sqrt{\left(D_{65}\right)\left(D_{35}\right)}$

Particle size at which 65 percent of the bed material by weight is finer

Particle size at which 84 percent of the bed material by weight is finer

Particle size at which 95 percent of the bed material by weight is finer

Base of natural logarithms

Weisbach friction factor

Acceleration due to gravity

Fraction of bed material in a given grain size

Fraction of bedload in a given size range

Fraction of suspended material in a given size range

Fraction of total load in a given size range

Rate at which a particle of diameter $D$ moves in suspension through a unit width per unit of time

Rate at which a particle of diameter $D$ moves through a unit width of the bed layer per unit of time

Rate at which a particle of diameter $D$ moves through a unit width of channel per unit of time

Sediment discharge through the bed layer of particles of a given size range, in tons per day 
$i_{T} Q_{T} \quad$ Total sediment discharge through the bed layer of particles of a given size range, in tons per day

$I_{1}$

$I_{2}$

$J_{1}$

$J_{2}$

$k$

$k_{s}$

K

$N_{s}$

$n$

$p$

$P$

$q_{B}$

$q_{s}$

$Q_{s}$

$Q_{s, M}$

$Q_{t s}$

$Q_{T M}$

$Q_{T C}$

$R$

$R^{\prime}$

$R_{B}$

(RS)

$S$

$S_{s}$

$S E$

$S E_{Q}$

$S E_{w}$

Temp

$v_{y}$

$v_{*}$

$v_{s}$

$V$

$V_{s}$

$V_{s r}$

$V_{s u}$

$V_{*}$

$V^{*}$

$W$

$W_{u}$

$w$

$X$

$x$

$x_{0}$

$y$

$Y$

$\boldsymbol{z}$

$\boldsymbol{z}_{r}$

A mathematical abbreviation

A mathematical abbreviation

A mathematical abbreviation

A mathematical abbreviation

Effective height of the roughness elements in a channel

Equivalent sand roughness for a particular roughness $k$

A constant at a particular time and cross section

Number of measurements in a set

Number of data sets for the particle-size range and data group.

Probability of a particle of a diameter $D$ being eroded

Mathematical abbreviation

Rate at which the bed layer moves through a unit width of cross section

Sediment load in suspension per unit of width, measured in weight, moving per unit of time between the water surface and the reference level $y=a$

Suspended sediment through a cross section, in tons per day

Measured suspended-sediment discharge

Sediment discharge in the sampled zone

Measured sediment discharge

Computed sediment discharge

Hydraulic radius

Hydraulic radius with respect to the grain

Hydraulic radius with respect to the grains on the bed

A hydraulic radius-slope parameter

Slope of the energy grade line

Specific gravity of sediment

Standard error of estimate

Square root of mean variance

Weighted approximate standard error of estimate

Water temperature in degrees Fahrenheit

Average point velocity at a distance $y$ from the closest boundary

Average shear velocity at the boundary; equals $\sqrt{\tau_{0} / \rho_{f}}$

Fall velocity for a sediment particle

Mean cross-sectional velocity of flow

Fall velocity for the geometric mean size for a size range

Fall velocity for the geometric mean size for a reference-size range

Fall velocity for the geometric mean size for a size range other than the range

Mean cross-sectional shear velocity; equals $\sqrt{g R S}$

Mean cross-sectional shear velocity with respect to grains on the bed

Weight of a particle

Underwater weight of a particle

Width of channel in feet

Einstein's characteristic grain size of a mixture

A dimensionless corrective factor

A variable; equals $k_{s} / k$

Distance from the streambed

Pressure correction in the transition from smooth to rough flow

A theoretical exponent of the equation that describes the vertical distribution of suspended sediment of a size range

$z$ for a reference size 
$z$ for size ranges other than that for the reference size

A logarithmic function

A logarithmic function

A logarithmic function

Universal constant characterizing turbulence

Thickness of laminar sublayer

$k_{s} / x$, the apparent roughness of a channel surface

$D_{65} / x$

Variability factor of lift; parameter varying with time which represents the fluctuation of velocity

Root-mean-square value of $\eta$

$\eta$ measured in $\eta$ values

Constant used to describe step movement of particles

Kinematic viscosity of water

"Hiding factor" for grains in a mixture

A correction factor

Density of fluid

Density of solids

Shear stress

Shear stress at the boundary

Intensity of transport

Intensity of transport for individual grain size for the Einstein procedure

Intensity of transport for individual grain size for the modified Einstein procedure

Intensity of shear on particles; function for correlating the effects of flow with the intensity of bedload transport

Intensity of shear on individual grain size

Intensity of shear on representative particle 


\title{
GENERAL STUDY OF THE MODIFIED EINSTEIN METHOD OF COMPUTING TOTAL SEDIMENT DISCHARGE
}

\author{
By D. E. Burkham and D. R. Dawdy
}

\section{ABSTRACT}

The modified Einstein procedure was revised by applying corrections to two equations and by replacing two equations. The revision was made because $D_{65}$ was used directly in the modified Einstein procedure to represent the equivalent sand roughness, $k_{s}$, whereas a much larger size apparently should have been used. For this study, 5.5 $D_{65}$, an average value based on a best fit for data for several sites, was used.

The revised modified Einstein procedure apparently is an improvement over the modified Einstein procedure. For the sediment and flow conditions at sites in five sand channels, the sediment discharges computed according to revised modified Einstein procedure apparently are not significantly more accurate than those computed according to modified Einstein procedure; however, improvement is indicated because the corrections of pertinent equations eliminates some of the empirical adjustments contained in the modified Einstein procedure. The revised procedure eliminated the need to arbitrarily divide the bedload transport intensity, $\phi_{*}$, by 2 which was done in the modified Einstein procedure. Values for $\phi_{*}$ are taken directly from a curve relating $\psi_{*}$ to $\phi_{*}$. The $\psi_{*}$ to $\phi_{*}$ relation was developed theoretically by Einstein, and it was verified by his experimental data. Values of $\phi_{*}$ determined from the $\psi_{*}$ to $\phi_{*}$ relation should be used directly in the Einstein bedload equation.

For the same data, the revised modified Einstein procedure gives shear velocities that are significantly larger and $x$ values that are significantly smaller than the corresponding values computed using the modified Einstein procedure. The ratio of shear velocity for the revised modified Einstein procedure to shear velocity for the modified Einstein procedure ranges from 9.52 to 1.28 for stream velocities ranging from 0.50 to 15 feet per second. For the 292 data sets used in testing the two procedures, the value of $x$ ranged from 1.00 to 1.14 for the revised modified Einstein procedure and from 1.00 to 1.62 , the maximum value possible, for the modified Einstein procedure. The value of $x$ is 1 for fully turbulent flow.

The approximate standard error of estimate for total sediment discharge determined for the five streams is about 22 percent for the two procedures. Except for the range for the largest particles tested, the weighted standard error is approximately equal in the two methods for the different sediment-size ranges. Weighted approximate standard errors for the different sediment-size ranges for the modified Einstein procedures and the revised modified Einstein procedures are: 17 percent and 17 percent for sizes smaller than $0.062 \mathrm{~mm} ; 25$ percent and 25 percent for sizes from 0.062 to $0.125 \mathrm{~mm} ; 46$ percent and 48 percent for sizes from 0.125 to $0.250 \mathrm{~mm}$; 72 percent and 77 percent for sizes 0.250 to $0.500 \mathrm{~mm}$; and 136 percent and 91 percent for sizes 0.500 to $1.00 \mathrm{~mm}$. The standard error for wash load is relatively low because the wash load is determined directly by sampling and the accuracy of values determined by sampling is better than those determined indirectly by equations, which is done for relatively large particles 
moving as bedload. The approximate standard error for total sediment discharge is only about 22 percent because a large fraction of the total sediment discharge was in suspension and the suspended-sediment discharge is determined directly by sampling. For a site on the Rio Grande conveyance channel, the amount of sediment is the size ranges of 0.062 to $0.125 \mathrm{~mm}, 0.125$ to $0.250 \mathrm{~mm}$, and 0.250 to $0.500 \mathrm{~mm}$ were significantly underestimated by the two procedures for periods when the bed form was flat or was in transition. Conversely, for these ranges of sediment sizes and for the total, the amount of sediment being moved was significantly overestimated for periods when the bed form was dunes.

For two sites on Fivemile Creek in Wyoming, the total sediment discharge is significantly overestimated. This statistic may be misleading. A sediment bed in motion at a normal section usually does not retain a fixed bed form for all flow conditions, therefore, the bed form at the sites on Fivemile River probably is dunes, flat, or in transition at different times. If so, the results found for the Rio Grande site probably apply for the other sites. The fact that the total sediment discharge is overestimated for a site may only indicate that a dune bed form was at the normal section during most of the periods when sediment transport data were being obtained.

\section{INTRODUCTION}

The development of a reliable method of computing sediment discharge at a site in a stream is one of the most important practical objectives of research in fluvial processes. The movement of sediment in an alluvial channel is complex, and because of this the rate of development of a reliable method has been slow. The engineer concerned with the movement of debris in a stream currently must rely strongly on experience and judgment in determining sediment discharge (American Society of Civil Engineers, 1975). Because of the expanding awareness of the effects of man on his environment, the need to develop better methods is increasing.

The U.S. Geological Survey is involved in research aimed at the development of new and reliable methods of measuring and computing sediment discharge. At the same time, it is attempting to improve methods that are already widely used. The study and the results described in this report are a part of the latter effort.

The modified Einstein method (Colby and Hembree, 1955) of computing total sediment discharge in open channels is widely used. The method is based on the excellent and original work of Einstein (1950). The MEP (modifed Einstein method) is an improvement over the original Einstein method; it is simpler in computation and it uses parameters more readily available from actual stream measurements. The modified method, however, contains empirical adjustments and it requires much experience and judgment in order to obtain reliable results.

The primary objective of this investigation was to apply theoretical corrections to pertinent equations used in the modified Einstein method and to determine if significant improvement results. Secon- 
dary objectives were to test the modified Einstein procedure for a wider range of flow and sediment conditions than had been previously applied, and to identify areas where additional research might be worthwhile. Two related criteria were used in the test for improvement resulting from the addition of the theoretical corrections. First, improvement is indicated if the accuracy of prediction for particle-size ranges and for total sediment discharge is improved. Second, improvement is indicated if the correction of pertinent equations eliminates some of the empirical adjustments contained in the method.

The study required extensive review of technical literature. The study procedure was as follows:

1. The basis for the theoretical correction was developed. This required a review of velocity and resistance equations developed by Keulegan (1938); Limerinos (1970); Leopold, Wilman, and Miller (1964); Einstein (1950); and Burkham and Dawdy (1976a). This part of the study is described in the section "Basis for Revision."

2. Pertinent equations and graphs used in the modified Einstein procedure for determining total sediment load were corrected. This required a review of the Einstein and modified Einstein procedures; identification of theoretical and empirical adjustments made by Einstein (1950) so equations and graphs for uniform particles would apply to mixtures; and identification of empirical adjustments made by Colby and Hembree (1955) to eliminate bias in the modified Einstein method. The review of the Einstein procedure is presented in the section "Einstein Method." The remainder of this part of the study is presented in the sections "Modified Einstein Procedure" and "Revised Modified Einstein Procedure."

3. Computer programs for MEP and RMEP (revised modified Einstein method) were developed. The computer programs are discussed briefly in the section "Computation Procedure."

4. Using test data, sediment discharges were computed and compared with measured values; comparisons were made by size ranges and for total sediment discharges. The standard errors of estimate for sediment discharges computed by the modified Einstein method were compared with the standard errors of estimate of discharges computed by the revised modified Einstein method to give an indication of improvement. The test data are for the following sites: Niobrara River near Cody, Nebr.; Middle Loup River at Dunning, Nebr.; Fivemile Creek near Riverton, Wyo.; Fivemile Creek near Shoshoni, Wyo.; and River Grande conveyance channel near Bernardo, N. M. Descriptions of this part of the study are presented in the sections "Comparison of Computed and Measured Sediment Discharges" and "Results of Comparison."

5. Finally, a summary and conclusions are presented. 


\section{BASIS FOR REVISION}

The Einstein and the modified Einstein methods (Einstein, 1950; Colby and Hembree, 1955) of computing total sediment discharge in open channels involve several equations and graphs that are based on Keulegan's (1938) solution of the Prandtl-von Kármán velocity distribution equation (Prandtl, 1926; von Kármán, 1930). The Keulegan (1938) equations are:

and

$$
v_{y}=v_{*}\left(8.5+5.75 \log y / k_{s}\right)
$$

in which

$$
V=V_{*}\left[(8.5-.9 b)+5.75 \log R / k_{s}\right]
$$

$v_{y}=$ average point velocity at a distance $y$ from the closest boundary;

$v_{*}=$ average shear velocity at the boundary; equals $\sqrt{\tau_{0} / \rho_{f}}$ where $\tau_{0}$ equals the shearing stress at the boundary and $\rho_{f}$ equals the density of the fluid;

$k_{s}=$ equivalent sand roughness for a particular roughness $k ; k$ is the effective height of the roughness elements in a channel;

$V=$ mean cross-sectional velocity of flow;

$V_{*}=$ mean cross-sectional shear velocity; equals $\sqrt{g R S}$;

$R=$ hydraulic radius;

$g=$ acceleration due to gravity;

$S=$ slope of the energy grade line;

$b=1$ divided by $\kappa$; and

$\kappa=$ universal constant characterizing turbulence; assumed to be 0.4 .

A general form of equation 2 is (Keulegan, 1938):

in which

$$
V=V_{*}[(C-.9 b)+5.75 \log (R / k)]
$$

$$
C=\text { a coefficient. }
$$

Equations 1 and 2 are applicable for fully developed turbulent flow in nonwavy rough uniform channels in which the resistance to flow is produced by the boundary surface. To use equations 1 and 2 , however, it is necessary to know the relation between the roughness factor, $k$, for a data set of interest and the equivalent sand roughness, $k_{s}$. A general equation relating $k$ and $k_{s}$ is (Keulegan, 1938):

$$
5.75 \log k_{s}=8.5-C+5.75 \log k
$$

Equation 3, according to Keulegan (1938), is the expression which gives the equivalent sand roughness $k_{s}$ for the particular roughness $k$. 
Physically, if a velocity $v$ is observed at a distance $y$ from a wall of arbitrary roughness $k$ under a known shear, the same velocity will be obtained at the same point and for the same shear if the particular roughness is replaced by closely packed uniform sand grains of roughness $k_{s}$. It is not practical to compute the equivalent sand roughness, $k_{s}$, using equation 3 , for every roughness height, $k$, found along the boundaries of uniform open channels. Instead, the effort traditionally has been to find a readily measurable parameter that could be used to approximate $k_{s}$ in equation 3 .

Equations $2 \mathrm{~A}$ and 3 can be combined to give a general equation that is applicable directly to turbulent flow in nonwavy rough uniform channels. Note that equation 3 can be rearranged to give:

$$
C=8.5-5.75 \log \left(k_{s} / k\right) \text {. }
$$

Equations 2 and $3 \mathrm{~A}$ are combined to give:

$$
V / V_{*}=\left(8.5-0.9 b-5.75 \log x_{0}\right)+5.75 \log (R / k)
$$

in which

$$
\begin{aligned}
b & =2.50, \text { and } \\
x_{\mathrm{o}} & =k_{s} / k .
\end{aligned}
$$

The term $x_{0}$ is introduced to simplify later discussions. Equation 4 is Keulegan's theoretical resistance equation for turbulent flow in nonwavy rough uniform open channels. Burkham and Dawdy (1976a) have developed a relation from which, by using equation $3 \mathrm{~A}, x_{0}$ can be approximated for a wide range of sediment sizes.

Empirically defined resistance equations by Limerinos (1970) and Leopold, Wolman, and Miller (1964) can be put in the form:

$$
1 / \sqrt{f}=C_{0}+2.03 \log (R / k)
$$

in which and

$f=$ Weisbach (1845) friction or resistance factor, equals $\frac{8 g D S}{V^{2}}$,

$$
C_{0}=\text { a constant. }
$$

The numerical value of the constant $C_{0}$, however, is different for the different equations. The parameters used to represent the distance from the boundary and used to represent roughness height also are different. Data for turbulent flow in nonwavy rough uniform channels were used by the different authors to develop the resistance equation having the form of equation 5 . 
Limerinos (1970) proposed that an equation using $D_{84}$ to represent roughness height, $k$, be used to compute resistance to flow in channels with coarse bed material. The equation is:

$$
1 \sqrt{f}=1.16+2.03 \log \left(R / D_{84}\right)
$$

in which

$D_{84}=$ particle size, referred to as intermediate diameter, that equals or exceeds that of 84 percent of the streambed particles by count; the sampling procedure was patterned after the Wolman (1954) method.

Using roughness values computed from equation 6, Limerinos (1970) determined the percentage difference between measured and computed roughness for each of 50 data sets; the standard deviation of the percentage differences amounted to 19 percent.

When Limerinos (1970) used $D_{50}$ to represent the roughness height, $k$, the equation was

$$
1 / \sqrt{f}=0.35+2.03 \log \left(R / D_{50}\right)
$$

For equation 7 Limerinos determined that the standard deviation of the percentage difference between measured and computed roughness amounted to 22.4 percent.

An equation in which $D_{16}$ is used to represent $k$ can be approximated directly from equation 6 and data contained in table 3 in Limerinos' (1970) report (Burkham and Dawdy, 1976a). The mean of the ratios, $D_{84} / D_{16}$, is 7.3 for the Limerinos data. If the factor $D_{84}$ in equation 6 is replaced with $7.3 D_{16}$, the resulting equation is

$$
1 / \sqrt{f}=-0.59+2.03 \log \left(R / D_{16}\right)
$$

Using the Limerinos data and the procedure outlined by Limerinos (1970), the standard deviation of the percentage difference for equation 8 was determined to be 27.6 percent.

Figure 1, developed using the Limerinos (1970) data, shows that the standard error of estimate apparently is significantly smaller when $D_{84}$ is used to represent the roughness height in the Keulegan equation than when $D_{50}$ or $D_{16}$ is used. Further improvement in accuracy might result if another larger size, say $D_{95}$, were used to represent the roughness height rather than $D_{84}$.

Burkham and Dawdy (1976a) used data contained in reports by Wolman (1954); by Leopold, Wolman, and Miller (1964); and by Barnes (1967) to verify equation (6) for turbulent flow in fairly rigid, 


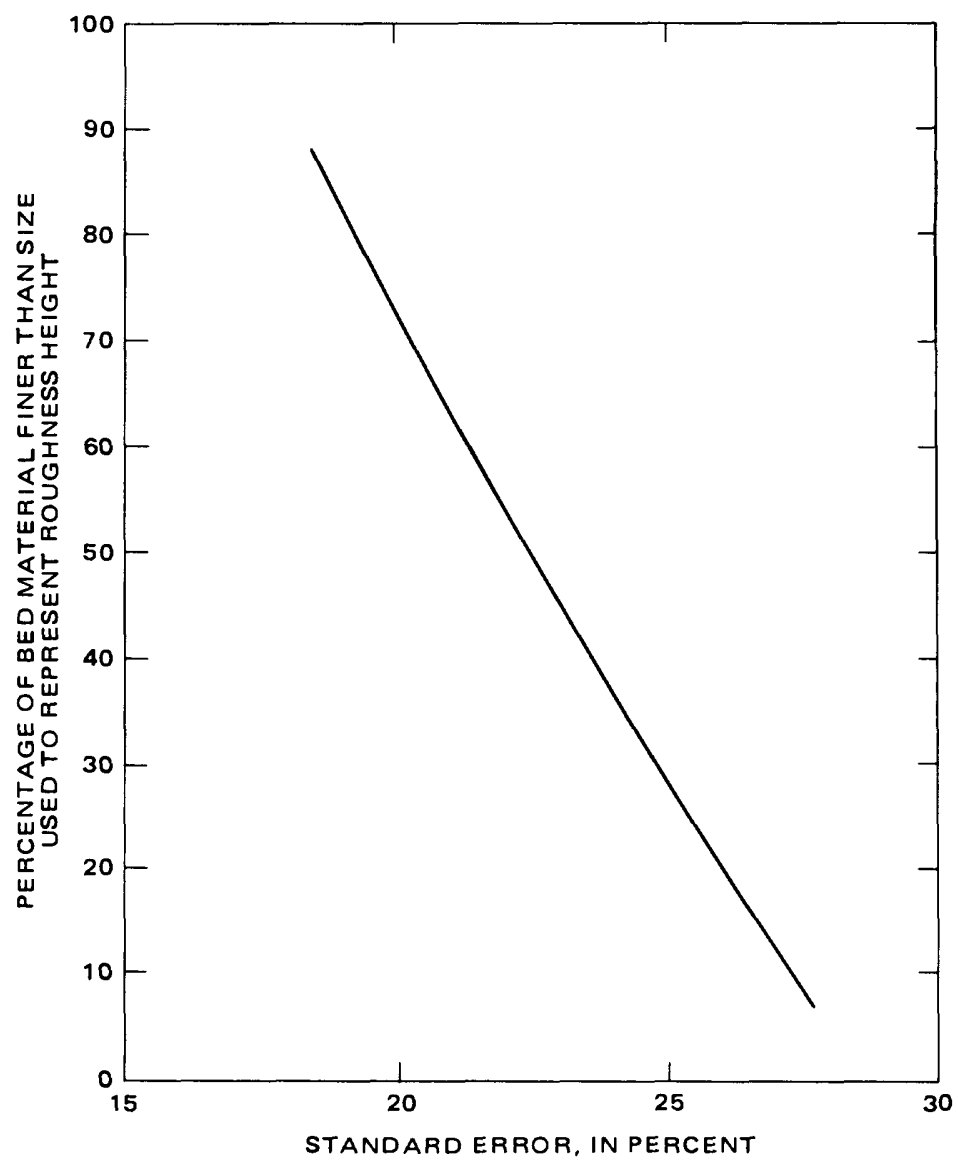

FigURE 1.-Relation between percentage of bed material finer than size used to represent roghness height, and standard error of estimate, in percent, for the different resistance equations for flow in rigid-boundary streams. (From Burkham and Dawdy, 1976a, fig. 3).

nonwavy channels (fig. 2). They used data contained in reports by Culbertson and Dawdy (1964), and by Dawdy (1961) to extend equation 6 to include upper-regime flow for sand-bed streams. As shown in figure 2, the resistance to upper-regime flow in sand-bed streams apparently is fairly well represented by the extension of equation 6 . The standard error of estimate for the sand-bed resistance for upperregime flow, computed using the Culbertson and Dawdy (1964) data and the Limerinos (1970) procedure, is about 13 percent (Burkham and Dawdy, 1976a). Because $1 / \sqrt{f=V /}(V * \sqrt{8})$, equation 5 can be changed to

$$
V / V_{*}=C_{1}+5.75 \log (R / k)
$$




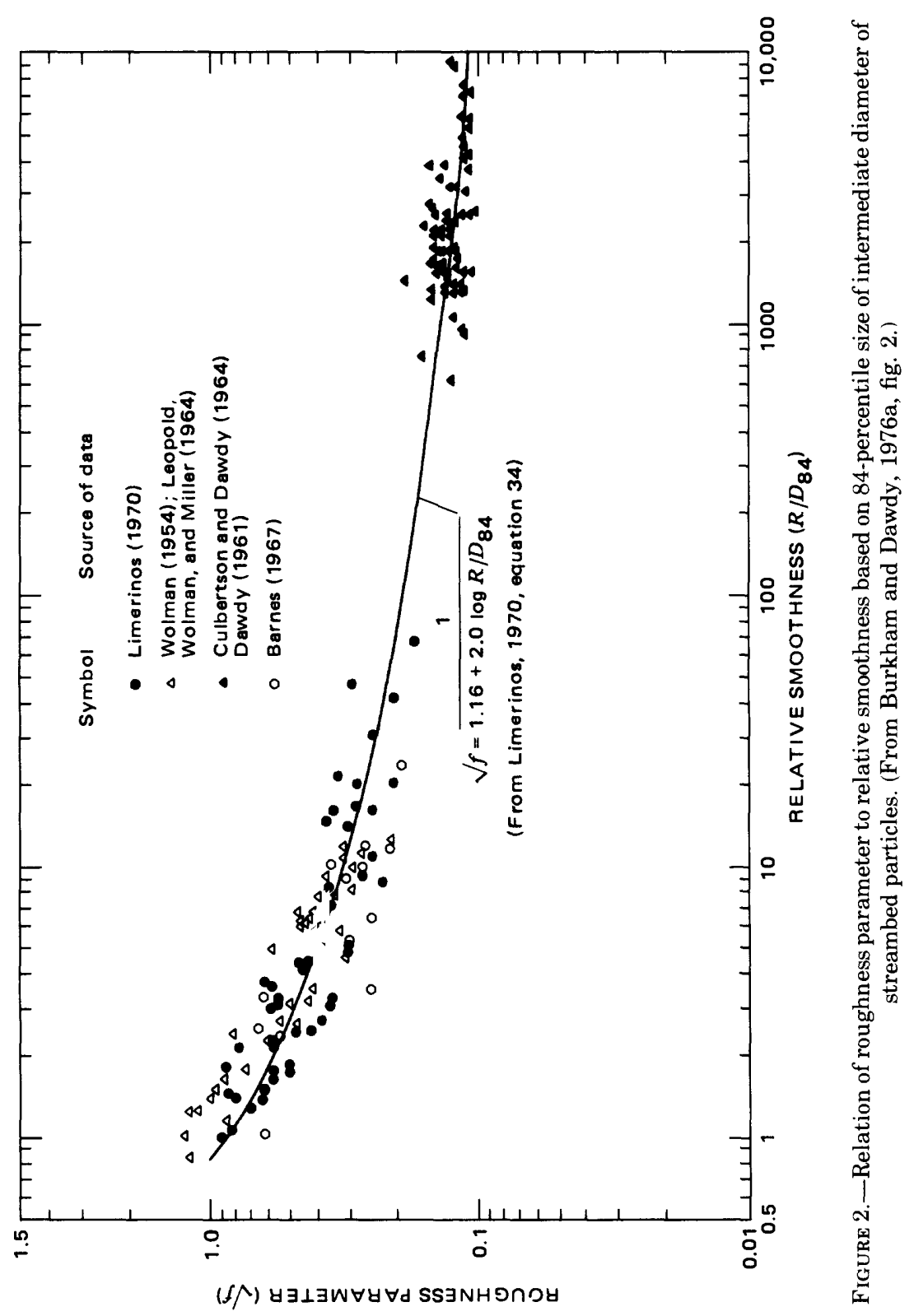


which is comparable directly with equation 4 . The different constants for equations $2 \mathrm{~A}, 4,5$ and 9 are related according to

$$
C_{1}=8.5-0.9 b-5.75 \log x_{0}=C-0.9 b=C_{0} / \sqrt{8}
$$

Equation 10 and data from reports by Limerinos (1970); by Dawdy (1961); by Leopold, Wolman, and Miller (1964); and by Barnes (1967) were the basis for the development of the curve shown in figure 3 (Burkham and Dawdy, 1976a). The curve shows the relation between percentage of bed material finer than the size used to represent roughness height and the coefficient $C$ in equation $2 \mathrm{~A}$.

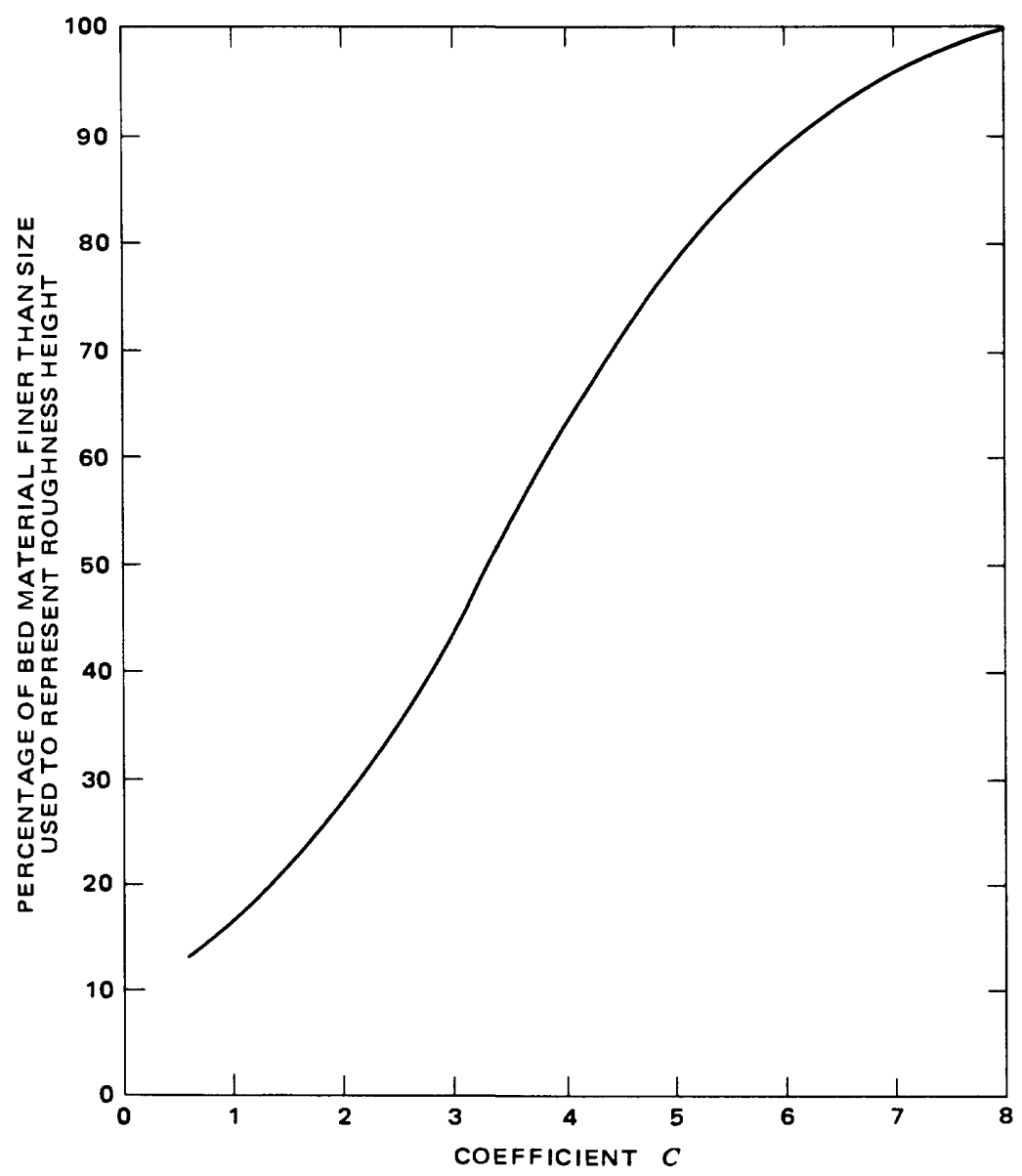

Figure 3.-Relation between percentage of bed material finer than size used to represent roughness height and the coefficient $C$ in the Keulegan equation: $V / V_{*}=$ $(C-0.9 b)+5.75 \log R / K$. (From Burkham and Dawdy, 1976a, fig. 3.) 
In the Einstein and the modified Einstein methods of computing total sediment discharge, $D_{65}$ is used directly to represent the roughness height, $k_{s}$, in equations developed from equations 1 and 2 (Einstein, 1950; Colby and Hembree, 1955); the correction factor -5.75 $\log x_{0}$, which equals $-5.75 \log k_{s} / D_{65}$ when $D_{65}$ is used to represent bed roughness, was not applied. The value of $x_{0}$ depends on the distribution of sediment-particle sizes in a mixture of interest; however, its value apparently decreases inversely with the percentile fractile used to represent channel roughness. On the basis of the relation in figure 3 and equation 10 , the average value of $x_{0}$ is about 5.5 when $D_{65}$ is used to represent the roughness height. The use of $D_{65}$ to directly represent roughness height in the Einstein and modified Einstein methods (Einstein, 1950; Colby and Hembree, 1955) of computing total sediment discharge probably introduced significant bias; empirical corrections are needed to eliminate this bias.

\section{EINSTEIN METHOD}

\section{INTRODUCTION}

The Einstein method is for computing total sediment discharge in the size ranges that are found in significant quantities in the streambed (Einstein, 1950). The computed total sediment discharge is the sum of the bedload discharge and the suspended-sediment discharge. The bedload discharge is considered to be through a bed layer two grain diameters thick, and the suspended sediment is considered to be discharge from the water surface to the bed layer.

Einstein's (1950) computations are for a representative cross section of a channel and an average energy slope. The area and wetted perimeter for a representative cross section are determined by averaging the areas and the wetted perimeters of a number of sections in a typical reach that is of sufficient length to permit an adequate definition of the overall energy slope. The theoretical basis for the Einstein method can be presented as (1) equations pertinent to suspended-sediment discharge, (2) equations pertinent to the bedload discharge, and ( 3 ) equations pertinent to the transition between bedload and suspended-sediment discharges.

\section{EQUATIONS PERTINENT TO SUSPENDED-SEDIMENT DISCHARGE}

Suspended-sediment discharge is computed as the integration of the product of the theoretical velocity and suspended-sediment concentration along a representative vertical in a cross section. Einstein (1950) combined equations developed by Keulegan (1938) to describe the theoretical velocity distribution in his representative vertical. 
The two equations are equation 1 and an equation to represent the velocity distribution for smooth boundaries which is

$$
v_{y} / V *=5.50+5.75 \log \frac{y V_{*}}{\nu}
$$

The results of combining equation 1 and 11 can be presented as

$$
v_{y} / V *=5.75 \log \left[30.2\left(\frac{y x}{k_{s}}\right)\right]=5.75 \log (30.2 y / \Delta)
$$

which represents the velocity distribution for smooth and rough conditions and the transition between smooth and rough conditions, where parameters in equation 11 and 12 that have not been defined previously are:

$\nu=$ kinematic viscosity of water;

$x=$ a dimensionless corrective factor; and

$\Delta=k_{s} x$, the apparent roughness of the channel surface.

The corrective factor $x$ is represented in figure 4 as a function of $k_{s} / \delta$ in which $\delta$ is the thickness of the sublayer of a smooth boundary; equals $\left(11.6 v / V_{*}\right)$. The $\left(k_{s} / \delta\right)$-to- $x$ correction curve was developed using equation 12 and the relation in Kuelegan's figure 3 (Kuelegan, 1938).

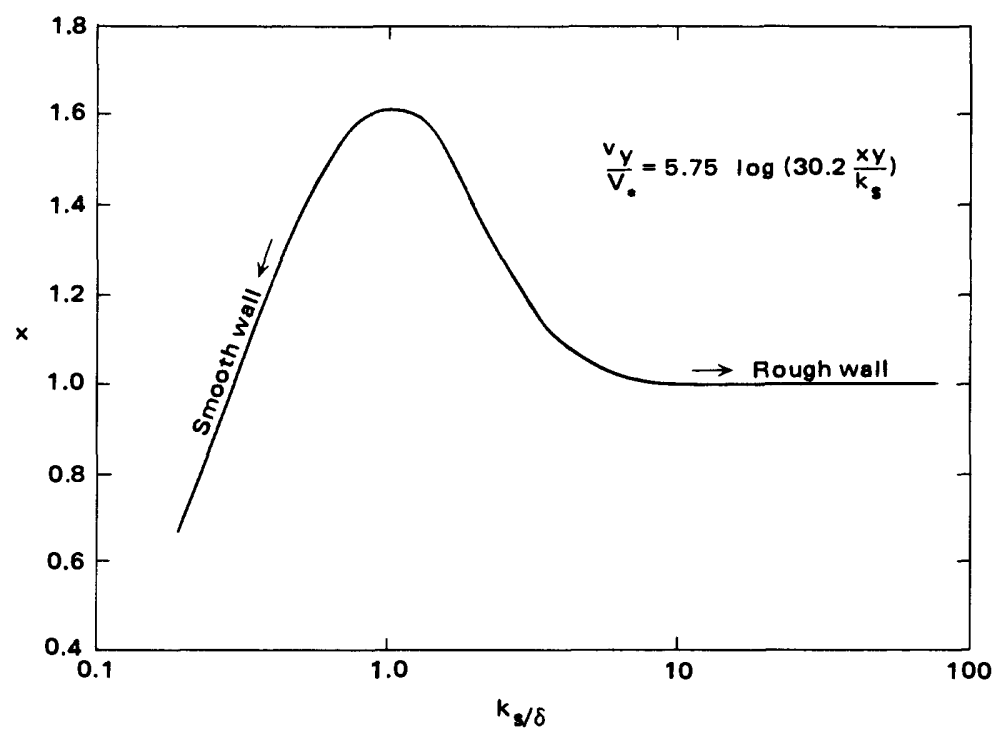

FIGURE 4.-Correction $x$ in equation 12 in terms of $k_{s} / \delta$. (From Einstein, 1950, fig. 4.) 
The suspended-sediment concentration in Einstein's (1950) representative vertical can be represented by:

in which

$$
C_{y} / C_{a}=\left[\left(\frac{d-y}{y}\right) \frac{a}{d-a}\right]_{z}^{z}
$$

$$
\begin{array}{cc}
C_{y}= & \text { sediment concentration for a particle having a diame- } \\
\text { ter, } D, \text { at a distance } y \text { above the streambed; } \\
C_{a}=\text { sediment concentration for a particle having a diame- } \\
\text { ter, } D, \text { at a distance } a \text { above the streambed; } \\
z=\text { an exponent. }
\end{array}
$$

Einstein (1950) used the formula $z=\left[v_{s} /\left(0.4 V_{*}\right)\right]$ to determine values for $z$, where $v_{s}$ represents the fall velocity for the particle.

An integral equation for the suspended load for a particle having a diameter, $D$, moving through a unit width of a cross section is obtained by combining equations 12 and 13 to give

or

$$
q_{s}=\int_{a}^{d} C_{y} v_{y} d y
$$

$$
q_{s}=\int_{y}^{d} C_{a}\left[\left(\frac{d-y}{y}\right) \frac{a}{d-a}\right]^{z} 5.75 V_{*} \log _{10}\left[\frac{(30.2 y x)}{k_{s}}\right] d y
$$

Einstein (1950) simplified equation 14 by (1) referring the concentration to that at the lower limits of integration $a$; (2) replacing $a$ with a dimensionless expression $A=a / d$; and (3) using $d$, mean depth of water, as the unit for $y$. The resulting equation to the base $\mathrm{e}$ is:

$$
\begin{aligned}
& q_{s}=5.75 V_{*} d C_{a}\left(\frac{A}{1-A}\right)^{z}\left[\log _{10}\left(\frac{30.2 d x}{k_{s}}\right)\right. \\
& \left.\int_{A}^{1}\left(\frac{1-y}{y}\right)^{z} d y+0.434 \int_{A}^{1}\left(\frac{1-y}{y}\right)^{z} \log _{e}(y) d y\right]
\end{aligned}
$$


in which

$q_{s}=$ the sediment load in suspension per unit of width, measured in weight, moving per unit of time between the water surface and the reference level $y=a$.

In order to present graphical solutions to the two integrals in equation 15, Einstein transformed equation 15 to:

$$
q_{s}=11.6 V_{*} C_{a} a\left\{2.0303 \log _{10}\left(\frac{30.2 d x}{k_{s}}\right)^{\left(I_{1}\right)+I_{2}}\right\}
$$

in which

$$
\begin{gathered}
I_{1}=0.216 \frac{(A)^{z-1}}{(1-A)^{z}} \int_{A}^{1}\left(\frac{1-y}{y}\right)^{z} d y \\
I_{2}=0.216 \frac{(A)^{z-1}}{(1-A)^{z}} \int_{A}^{1}\left(\frac{1-y}{y}\right)^{z} \log _{e}(y) d y
\end{gathered}
$$

According to Einstein (1950), the product, $11.6 V_{*}$, is the flow velocity at the outer edge of the laminar sublayer in the case of a hydraulically smooth bed, or the velocity in a distance of 3.68 roughness diameters from the bed in case of a rough wall. The functions $I_{1}$ and $I_{2}$ in terms of $A$ and $z$ are shown in Einstein (1950, figs. 1 and 2), Colby and Henbree (1955, figs. 4 and 7), and Hubbell and Matejka (1959, figs. 11 and 13).

Equation 16 represents Einstein's (1950) theoretical basis for estimating suspended discharge per unit width for a size of sediment found in appreciable amounts in the bed. It does not consider wash load which consists of particles too small to be in the bed in appreciable amounts, nor is it applicable for computing sediment movement in the bed layer in which suspension is impossible.

Equations 1, 2, 11, and 12 are applicable for fully developed turbulent flow in nonwavy, rough, uniform channels; the resistance to flow is due to grain roughness only. Einstein's (1950) rationale for using equations $1,2,11$, and 12 to develop a method of estimating sediment transport in sand channels having significant bed forms is based on the assumption that the energy of turbulence corresponding to shape resistance does not contribute significantly to bedload motion and may be largely neglected in the entire sediment picture. 


\section{EQUATIONS PERTINENT TO BEDLOAD}

The quantity of sediment transported in a stream by rolling, sliding, or skipping within the bed layer is called the bedload. Einstein's (1950) theory for the rate of movement and concentration in the bed layer is based on the probability that a given particle will move from its position in the streambed and that the position in the bed will be occupied by another particle. By assuming that the bed is neither eroding nor scouring, Einstein (1950) developed a bedload equation that can be represented as:

in which

$$
i_{B} q_{B}=\phi_{*} i_{b} \rho_{s} g^{3 / 2} D^{3 / 2}\left(S_{s}-1\right)^{1 / 2}
$$

$i_{B} q_{B}=$ the rate at which a particle of diameter $D$ in a mixture moves through a unit width of the bed layer per unit of time; $i_{B}$ is the fraction of bedload in a given size range; $q_{B}$ is the rate at which the bedlayer sediment moves through the unit width of cross section;

$\phi_{*}=$ intensity of transport for an individual grain size;

$i_{b}=$ fraction of bed material in a given grain size;

$\rho_{s}=$ density of the solids and

$S_{s}=$ specific gravity of the sediment.

For a given particle of diameter $D$, Einstein assumed that all parameters on the right side of equation 19 are constant except for $\phi_{*}$. The intensity of transport, $\phi_{*}$, is a function of the intensity of shear, $\psi_{*}$, for the individual grain size. A curve relating $\psi_{*}$ and $\phi_{*}$ given in Einstein (1950 fig. 10), developed by Einstein (1950, p. 37, eq. 57) is entirely theoretical but was verified by experimental data. The numerical values of the constants used by Einstein (1950) to develop the curve are:

$$
\begin{aligned}
A_{*} & =1 / 0.023=43.5 \\
B_{*} & =1 / 7=0.143 \\
\eta_{0} & =1 / 2=0.5 .
\end{aligned}
$$

The numerical values for the constants $A_{*}$ and $B_{*}$ were based on results of bedload experiments with uniform grains; $\eta_{o}$ is an assumed value.

The intensity of shear, $\psi$, for uniform sand, and fully turbulent flow is determined from;

$$
\Psi=\frac{\rho_{s}-\rho_{f}}{\rho_{f}}\left(\frac{D}{R_{B}{ }_{B} S}\right)
$$

in which

$R_{B}^{\prime}=$ hydraulic radius with respect to the grains on the bed. Einstein's (1950) reason for using $R^{\prime}{ }_{B}$ instead of $R$ is based on the 
hypothesis that the energy of moving water is dissipated as friction in two ways: (1) along sediment grains of the surface as a rough wall with the representative grain diameter equal to $k_{s}$; and (2) by separation of the flow from the surface at characteristic points of the ripples or bars. He assumed that the energy dissipation occurring at the sediment grains creates turbulence in the bed layer that has a great effect on bedload movement; this is the energy dissipation that must be accounted for in developing bedload equations. The term $R_{B}{ }_{B}$ is used to represent the hydraulic radius with respect to the grains on the bed, and the expression $V^{\prime}{ }_{*}=\sqrt{g R^{\prime}{ }_{B} S}$ is used to refer to the shear velocity with respect to the grains on the bed.

Einstein (1950) developed correction parameters which could be used in conjunction with equation 20 to estimate values of $\psi_{*}$ for particles in a mixture that were based on the following assumptions and criteria:

1. The velocity acting on all particles of a mixture must be measured at a distance $0.35 D$ from the theoretical bed, where:

$$
\begin{aligned}
& X=0.77 \Delta \text { if } \Delta / \delta>1.80: \\
& X=1.39 \delta \text { if } \Delta / \delta<1.80:
\end{aligned}
$$

2. The particles smaller than $X(X>D)$ seem to hide between the other particles or in the laminar sublayer, respectively, and their lift must thus be corrected by division with a parameter $\xi$ which itself is a function of $D / X . X$ is Einstein's characteristic grain size of a mixture.

3. An additional correction factor $Y$ was found to describe the change of the lift coefficient in mixtures with various roughness conditions.

Einstein's (1950) equation for determining $\psi_{*}$ for particles in a mixture is:

in which

$$
\Psi_{*}=\xi Y\left(\beta^{2} / \beta_{x}{ }^{2}\right) \frac{\rho_{s}-\rho_{f}}{\rho_{f}} \frac{D}{R_{B}^{\prime} S}
$$

$$
\begin{aligned}
& \beta=\log (10.6) \\
& \beta_{x}=\log (10.6 X / \Delta) ; \\
& \Delta=D_{6.5} / x ; \text { and }
\end{aligned}
$$

where

$\xi$ is a function of $D / X$ which is shown in Einstein (1950, fig. 7) and

$Y$ is a function of $D_{65} / \delta$ which is shown in Einstein (1950, fig. 8). The empirical relations shown in Einstein (1950, figs. 7 and 8) were based on flume experiments.

\section{EQUATIONS FOR TRANSITION BETWEEN BEDLOAD AND SUSPENDED LOAD}

Einstein's (1950) equation relating sediment transported as bedload to that in suspension for all particle sizes for which a bedload 
function exists can be represented as.

$$
i_{s} q_{s}=i_{B} q_{B}\left\{P I_{1}+I_{2}\right\}
$$

in which

$$
P=\left(\frac{1}{0.434}\right) \log \left(\frac{30.2 d x}{k_{s}}\right)=2.303 \log \left(\frac{30.2 d x}{k_{s}}\right)
$$

Equation 22 was developed from equation 16 and an assumption that $q_{B}$ can be represented by the expression $q_{B}=11.6 V_{*} C_{a} a$. The total load, $i_{T} q_{T}$, can be represented by

$$
i_{T} q_{T}=i_{B} q_{B}+i_{s} q_{s}=i_{B} q_{B}\left(P I_{1}+I_{2}+1\right) .
$$

\section{MODIFIED EINSTEIN PROCEDURE}

\section{INTRODUCTION}

The modified Einstein procedure (Colby and Hembre, 1955) of computing total load for the ranges of large particle sizes differs from that used for the ranges of small particle sizes. In the ranges of the large particles-ranges for the reference size and larger sizes (Colby and Hembree, 1955), the Einstein procedure is used except that different methods of computation are used for the shear with respect to the sediment particles, $\psi_{*}$; the intensity of the bedload transport, $\phi_{*}$; and the exponent $z$. For ranges smaller than that for the reference size, the sediment discharge is obtained by multiplying the suspendedsediment discharge in the sampled zone by a ratio determined on the basis of equation 16 .

\section{EQUATIONS FOR RANGES OF LARGE PARTICLES}

Colby and Hembree (1955) used a modified version of equation 24 to compute the total discharge of sediment in the ranges of large particles. The modified equation is:

$$
\left(i_{T} Q_{T}\right)_{M}=\left(i_{B} Q_{B}\right)_{M}\left(P I_{1}+I_{2}+1\right)
$$

in which

$\left(i_{T} Q_{T}\right)_{M}=$ total sediment discharge through a cross section for a given range of particle sizes, in tons per day; 
$\left.i_{B} Q_{B}\right)_{M}=$ sediment discharge through the bed layer of particles of the given size range, in tons per day; equals $\left(i_{B} q_{B}\right)_{M}$ times $43.2 w$;

where

$\left(i_{b} q_{b}\right)_{M}=$ sediment discharge through the bed layer of particles of the given size range, in pounds per second per foot of width; and $w=$ width of channel in feet.

The subscript $M$ denotes a parameter that was obtained according to MEP. The 43.2 is a constant for converting pounds per second to tons per day.

The MEP equation for determining $\left(i_{B} Q_{B}\right)_{M}$ is based on equation 19. The value of the intensity of the bedload transport $\psi_{* M}$, used in entering the relation (See Einstein (1950, fig. 10)), however, is based on $D_{35}$ and $D_{65}$; and the value of $\phi_{*}$ obtained from the relation is arbitrarily divided by 2 (Colby and Hembree, 1955). The MEP equation for bedlayer discharge in a cross section of a stream is

$$
\left(i_{B} Q_{B}\right)_{M}=\phi_{* M} 43.2 w 1,200 i_{b} D^{3 / 2}
$$

in which

$$
\phi_{* M}=\phi_{*} / 2
$$

The 1,200 represents the constants in equation 19 in which $g$ is the gravitational constant, $32.2 ; \rho_{s}$ equals 5.17 and $S_{S}$ equals 2.65 (Einstein, 1950). The quantity $\psi_{* M}$ is computed using the equations:

$$
\begin{gathered}
\psi_{* M}=\left(1.65 D_{35}\right) /(R S)_{M} ; \text { and } \\
\psi_{* M}=(0.66 D) /(R S)_{M} ;
\end{gathered}
$$

and the larger $\psi_{* M}$ from these equations is used for each geometric mean particle size for a range of sizes. The slope-hydraulic radius parameter, $(R S)_{M}$, is obtained by using Einstein's (1950) equation 9 except that the average depth, $d$, replaces $R^{\prime} ; D_{65}$ is used to represent $k_{s}$. Einstein's (1950) equation 9 was derived by combining a Keulegan velocity equation for a hydraulically smooth bed with another Keulegan velocity equation for a hydraulically rough bed. The MEP velocity equation is

$$
V / V_{* M}=5.75 \log \left(12.27 \frac{d x}{D_{65}}\right)
$$

in which

$$
V_{* M}=\sqrt{g(R S)_{M}}
$$


A value for $(R S)_{M}$ is obtained by solving equation 29 and 30 from a known value for $V$ and where $x$ is the same function of $k_{s} / \delta_{M}$ as given in figure 4 and

$$
\delta=\frac{11.6 \nu}{V_{* M}}
$$

Solution of equation 29 and 30 to obtain a value for $V_{* M}$ is by trial with $x$ obtained from figure 4 .

A value for $z$ must be determined before the sum of $\left(P I_{1}{ }^{\prime \prime}+I_{2}{ }^{\prime \prime}+1\right)$ can be evaluated for a size range of large particles. Colby and Hembree (1955) combined equations 16, 22 and 24 to develop an equation for determining $z$. They reasoned that, for a given time at a cross section of a stream, equation 16 can be presented as:

$$
q_{s}=K\left(P J_{1}+J_{2}\right)
$$

and

$$
q_{s}{ }^{\prime} / q_{s}{ }^{\prime \prime}=Q_{s}{ }^{\prime} / Q_{s}{ }^{\prime \prime}=\frac{P J_{1}{ }^{\prime}+J_{2}{ }^{\prime}}{P J_{1}{ }^{\prime}+J_{2}{ }^{\prime \prime}}
$$

or

in which

$$
Q_{s}{ }^{\prime \prime}=Q_{s}{ }^{\prime}\left[\frac{P J_{1}{ }^{\prime \prime}+J_{2}{ }^{\prime \prime}}{P J_{1}{ }^{\prime}+J_{2}{ }^{\prime}}\right]
$$

$Q_{s}=$ suspended sediment through the cross section, in tons per day, for a given range of particle sizes;

$$
\begin{aligned}
& J_{1}=\int_{A}^{1}\left(\frac{1-y}{y}\right)^{z} d y \\
& J_{2}=\int_{A}^{1}\left(\frac{1-y}{y}\right)^{z} \log _{e}(y) d y
\end{aligned}
$$

$K=$ a constant at a particular time and cross section; and

$$
P=2.303 \log \left(\frac{30.2 d x}{D_{65}}\right)
$$

A single prime mark designates a symbol that is associated with sampling depth and a double prime mark designates a symbol that is 
associated with the total depth through which suspended sediment is discharged. The function $J_{1}$ and $J_{2}$ in terms of $A$ and $z$ are presented in Colby and Hembree (1955, figs. 5 and 6) and Hubbell and Matejka (1959, figs. 9 and 10). Equation 22 can be put in the form

$$
Q_{s}{ }^{\prime \prime}=i_{B} Q_{B}\left(P I_{1}{ }^{\prime \prime}+I_{2}{ }^{\prime \prime}\right)
$$

which can be combined with equation 34 to give:

$$
\frac{Q_{s}{ }^{\prime}}{i_{B} Q_{B}}=\frac{I_{1}{ }^{\prime \prime}}{J_{1}{ }^{\prime \prime}}\left(P J_{1}{ }^{\prime}+J_{2}{ }^{\prime}\right)
$$

The parameters $Q_{s}{ }^{\prime}$ and $i_{B} Q_{B}$ are computed independently of $z\left(Q_{s}{ }^{\prime}\right.$ is determined from measurements, and $i_{B} Q_{B}$ is determined using equation 26). Equation 39 is used to compute $z$ by trial for the reference size, the geometric mean size for a size range that is represented in appreciable quantities in both bedload discharge and suspendedsediment discharge. The $z$ 's for the other size ranges are computed by proportion from the $z$ for the reference-size range by using:

$$
z_{u}=z_{r}\left(\frac{V_{s u}}{V_{s r}}\right)^{0.7}
$$

in which

$z_{u}=z$ for size ranges other than that for the reference size; the unknown;

$z_{r}=z$ for reference size;

$V_{s u}=$ fall velocity of the geometric mean size for the other size ranges; and

$V_{s r}=$ fall velocity of the geometric mean size for the reference size.

\section{EQUATIONS FOR RANGES OF SMALL PARTICLES}

Equation 34 is used to compute total suspended-sediment discharge for ranges of particle sizes smaller than the range for the reference size. The $z$ 's for the different ranges of sizes are determined using $z$ for the reference size in equation 39 . The suspended-sediment discharge for a range of particle sizes obtained using equation 34 normally represents the total sediment discharge for the range; if $i_{B} Q_{B}$ is significant for the range, however, it must be added.

\section{REVISED MODIFIED EINSTEIN PROCEDURE}

The RMEP (revised modified Einstein procedure) is similar to the MEP except several equations have been changed. Equations for shear velocity, bedload, exponent $z$, and total load are affected. 


\section{SHEAR VELOCITY}

The equation used to compute shear velocity is derived from equation 29 by replacing $D_{65}$ with $5.5 D_{65}$ (Burkham and Dawdy, 1976a) to give

$$
V_{* R M}=V /\left(5.75 \log \left[2.23 \frac{d x}{D_{65}}\right]\right)
$$

Values for $V_{* R M}$ are obtained by using known values for $V, d$ and $D_{65}$; and trial values of $x$ in equation 41 . Values for $(R S)_{R M}$ are obtained using the equation

$$
(R S)_{R M}=\left(\frac{V_{* R M}}{g}\right)^{2}
$$

The curve in figure 4 is assumed to be applicable when $k_{s} / \delta$ is represented by $5.5 D_{65} / \delta_{R M}$ and $\delta_{R M}$ is obtained using the expression $\delta_{R M}=$ $11.6 \mathrm{v} /\left(V_{*}\right)_{R M}$. The change in the representative size from $D_{65}$ as used in the modified Einstein method to $5.5 D_{65}$ is based on results which indicate that the $k_{s}$ used in the original Einstein development is a size fraction much larger than $D_{65}$, for which $5.5 D_{65}$ was found to be an average value (Burkham and Dawdy, 1976b). The subscript $R M$ denotes a parameter that was obtained according to RMEP.

The shear velocity obtained according to RMEP is significantly larger than the shear velocity obtained according to MEP. The relation between the two shear velocities can be represented by:

$$
(g R S)_{R M}^{1 / 2}=\left(1+\frac{4.26}{V}\right)(g R S)_{M}^{1 / 2}
$$

The ratio $(g R S)_{R M}^{1 / 2} /(g R S)^{1 / 2}{ }_{M}$ ranges from 9.52 to 1.28 for velocities ranging from 0.50 to 15 feet per second.

Equation 41 is assumed to be valid for turbulent flow in open channels where the turbulence results totally from grain roughness. The rationale for using equation 41 to determine shear velocity in channels having significant bed form is based on Einstein's (1950) assumption that the energy of turbulence generated because of shape resistance does not contribute significantly to bedload motion and may be largely neglected in the entire sediment picture. Given a velocity, a depth, and a value for $D_{65}$ for a site in a stream having significant form roughness, $V_{* R M}$ would approximately represent the shear velocity that would have occurred if no form roughness had existed at the site.

Colby (1961) has shown that sediment transport for sand-channel streams is primarily related to velocity of flow, approximately to the 
third power. Change of regime alters the form resistance and the velocity of flow, but the difference in transport is directly linked to the change in velocity. This tends to support Einstein's assumption that an effective shear computed without directly considering form roughness should be used to estimate transport of sand for all regimes of flow.

\section{BEDLOAD}

Equation 19 and the relation in Einstein (1950, fig. 10) are used in the RMEP to determine bedload. However, the procedure used to derive a value for the intensity of shear, $\left(\psi_{*}\right)_{R M}$, which is needed to use the $\psi_{*}$-to- $\phi_{*}$ relation, is different from the procedures used by Einstein (1950) and by Colby and Hembree (1955). The approach used in developing an equation to represent $\left(\psi_{*}\right)_{R M}$ was indirect, included several assumptions, and involved several steps. A general equation for $\psi_{*(R, M)}$ was developed by first developing an equation for a uniform particle of size $k_{s}$ and then applying corrections to this equation so that it would be applicable to mixtures. The equation for the uniform particle is

$$
\Psi_{*(R M)}=1.65 \frac{k_{s}}{(R S)_{R M}}
$$

which was obtained by using Einstein's (1950) equation 49; replacing $D$ with $k_{s}$ and $\left(R^{\prime} S\right)$ with $(R S)_{R M}$ and representing $\rho_{s}-\rho_{f}$ with 1.65 .

The general equation for $\psi_{* R M}$ for a mixture is

$$
\Psi_{* R M}=1.65(\xi Y)_{R M}\left(\beta / \beta_{x}\right)^{2} \frac{k_{s}}{(R S)_{R M}}
$$

in which

$$
\begin{aligned}
& \beta=\log (10.6) ; \text { and } \\
& \beta_{x_{1}}=\log (10.6 x) .
\end{aligned}
$$

In developing equation 45 a basic assumption was made that Einstein's hiding coefficient and lift correction can be represented by the single parameter $(\xi Y)_{R M}$.

Burkham and Dawdy (1976a) found that $x_{0}$ is approximately 1 when channel roughness in a hydraulically rough channel is represented by a relatively large particle in a mixture of sizes. For a hydraulically rough channel, $k_{s}$, therefore, is assumed to be equal to the diameter, $D$, for relatively large particles in a mixture and $(\xi Y)_{R M}$ is assumed to be 1 for the relatively large particles in a mixture. Considering these assumptions, $\psi_{* R M}$ for relatively large particles in a mixture in a hydraulically rough channel can be represented as

$$
\psi_{* R M}=1.65\left(\beta / \beta_{x_{1}}\right)_{2} D /(R S)_{R M}
$$


For the RMEP, $\psi_{* R M}$ from equation 46 is assumed to represent the largest value for the intensity of shear that is possible for a particle of size $D$ in a mixture; the value of $\phi_{* R M}$, obtained by using a value of $\psi_{* R M}$ from equation 46 and the curve in Einstein (1950, fig. 10), would represent the lowest possible intensity of transport.

According to Einstein, $\xi$ is a function of $\delta, D_{65}, D$ and $X$; and $Y$ is a function of $\delta$ and $D_{65}$. For the current study, $(\xi Y)_{R M}$ is assumed to be a function of $\delta_{R M}, D_{65}, D_{35}, D$ and $k_{s}$ as represented by the equation;

$$
(\xi Y)_{R M}=\left(\delta_{R M}+C_{s} D\right) / k_{s}
$$

in which

$C_{s}=$ a variable coefficient which is a function of the median diameter of the bed material (the geometric mean of $D_{35}$ and $D_{65}$ was used to represent the median diameter).

Equations 45 and 47 are combined to give

$$
\left(\psi_{*}\right)_{R M}=1.65\left(\beta / \beta_{x}\right)^{2}\left(\delta_{R M}+C_{s} D\right) /(R S)_{R M}
$$

which is used to compute $\left(\psi_{*}\right)_{R M}$ for all ranges of particle sizes where $\left(\delta_{R M}+C_{s} D\right)$ is larger than the geometric mean diameter, $D$; equation 46 is used when $D$ is equal to or greater than $\left(\delta_{R M}+C_{s} D\right)$. Values of $\phi_{*}$ obtained by entering $\left(\psi_{*}\right)_{R M}$ in the relation shown in Einstein (1950, fig. 10) are used directly in equation 19 to compute bedload.

The empirically defined equation for $C_{s}$ is

$$
C_{s}=-0.62+3.12\left[\left(D_{65}\right)\left(D_{35}\right)\right]^{1 / 2}
$$

which is applicable in the range $0<C_{s}<1$. The coefficient $C_{s}$ is zero when $D_{50 \mathrm{G}}{ }^{-}$equals $\left[\left(D_{65}\right)\left(D_{35}\right)\right]^{1 / 2}-$ is smaller than about $0.2 \mathrm{~mm} ; C_{s}$ is 1 when $D_{50 G}$ is 0.52 or larger. The development of equation 49 is described in the section "Computation Procedure."

\section{EXPONENT $Z$ AND TOTAL LOAD}

The RMEP uses equation 39 with RM values inserted for the trial determination of $z$. The value of $P_{R M}$ used in equation 39 is determined from equation 50 which is

$$
P_{R M}=2.303 \log \left(\frac{30.2 d x}{5.5 D_{65}}\right)=2.303 \log \left(\frac{5.5 d x}{D_{65}}\right)
$$

The total discharge of sediment in the range of large particle sizes is then computed as in equation 25 , but with RMEP parameters in the equation:

$$
i_{t} Q_{t}=\left(i_{B} Q_{B}\right)_{R M}\left(\left(P_{R M}\right) I_{1}^{\prime \prime}+I_{2} "+1\right)
$$


Similarly, equation 34 for the ranges of small sediment sizes is revised to

$$
i_{t} Q_{t}=Q_{s}{ }^{\prime \prime}=Q_{s}{ }^{\prime}\left[\frac{P_{R M} J_{1}{ }^{\prime \prime}+J_{2}{ }^{\prime \prime}}{P_{R M} J_{1}{ }^{\prime}+J_{2}{ }^{\prime}}\right]
$$

\section{COMPARISON OF COMPUTED AND MEASURED SEDIMENT DISCHARGES}

BASIC DATA

Data sets for selected section in five river reaches are used in the comparison studies. The study sites are: Niobrara River at Cody, Nebr.; Middle Loup River near Dunning, Nebr.; Fivemile Creek at Shoshoni, Wyo.; Fivemile Creek near Riverton, Wyo.; and Rio Grande conveyance channel near Bernardo, N. M. In each reach the total sediment discharge was determined by sampling at a natural or manmade turbulence flume and data pertinent to the computation of total discharge were collected at nearby normal or natural sections. The total sediment discharge determined by sampling at the turbulence flume was taken to be total measured sediment discharge.

\section{NIOBRARA DATA}

The data, the flume, and the normal sections for the reach along the Niobrara River near Cody, Nebr. are described in a report by Colby and Hembree (1955). The data were used by them in the development of th MEP and in comparison of measured and computed total sediment discharges. They made comparisons for 24 sets of data, and these comparisons are summarized in their table 34 . Only 21 of the 24 data sets, however, were used in the current study. According to Colby and Hembree (1955), two data sets for the normal sections contained "obviously incorrect suspended-sediment size distribution." These two sets of data were not used in the current study. For a third set, Colby and Hembree made "separate computations for each of the two parts of the cross section" in computing total sediment discharge for the normal section. The third set was not used in the current study because of the uncertainty of what cross-sectional properties should be used in computing total sediment discharge for the normal section. Of the remaining 21 sets, some of the data used by Colby and Hembree-stream width, depths, and velocity; concentration of suspended sediment; size distribution of suspended sediment; average depth at the verticals where the suspended-sediment samples were collected; size distribution of bed material; and water temperaturecould not be duplicated exactly. The values of sediment discharge 
computed for this study using the modified Einstein procedure, therefore, do not agree exactly with those contained in table 34 in the report by Colby and Hembree.

\section{MIDDLE LOUP RIVER DATA}

The data, the flume, and the normal sections for the reach along the Middle Loup River at Dunning, Nebr., are described in three reports. The flume, the normal sections, and the 46 sets of data for March 1950 to September 1952 are described in a report by Hubbell and Matejka (1959); the 6 sets of data for November 1955 to March 1956 are described in a report by Hubbell, Jordan, Culbertson, and Hembree (1956); and the 7 sets of data for August 6, 1956, to November 30, 1956, are described in a report by Hubbell (1960).

Hubbell and Matejka (1959, table 8) made comparisons of measured and computed total sediment discharges in which the MEP was used in determining computed values for 63 sets of data for the study reach of the Middle Loup River. According to Hubbell and Matejka (1959), the following information from each section was used for the computations of total sediment discharge:

The average bed-material size distribution that was determined from all available samples, other than special samples, collected at the section; the measured suspendedsediment concentration and size distribution; the measured sediment discharge computed from the rated water discharge, except when the load was computed by parts; multipliers based on fall velocities from Rubey's equation. Most of the computed total loads for section $A$ and some of the computed total loads for section $E$ were determined from individual computations for each of two parts or more of the section...

The data sets for section $A$ and some for section $E$ were not used in the current study because of the uncertainty of what cross-sectional properties should be used in computing total sediment discharges. Of the remaining 46 data sets, some of the data used by Hubbell and Matejka could not be duplicated exactly. The values of sediment discharge computed for this study using the MEP, therefore, do not agree exactly with those contained in the report by Hubbell and Matejka (1959).

Comparisons made by Hubbell, Jordan, Culbertson, and Hembree (1956) are shown in their table 13 and comparisons made by Hubbell (1960) are shown in his table 5. For these two tables, the computed values for each data set were obtained using the MEP.

\section{FIVEMILE CREEK DATA}

The flumes and the normal sections for the reaches along the Fivemile Creek near Shoshoni and near Riverton, Wyo., are described briefly in a report by Colby, Hembree, and Rainwater (1956). The 87 sets of data for September 1954 to June 1, 1963, for the Shoshoni 
reach and the 64 sets of data for August 26, 1954, to September 1963 for the Riverton reach were copied directly from records in the files of the U.S. Geological Survey, Cheyenne, Wyo. Data for measuredsediment discharge for the different sediment-size ranges were included in 12 of the sets of data for the Shoshoni reach and in 14 for the Riverton reach.

\section{RIO GRANDE REACH}

The flumes, the normal sections, and the data for the reach along the Rio Grande conveyance channel near Bernardo, N. M., are described in reports by Harris and Richardson (1964); Gonzalez, Scott, and Culbertson, (1969); and Culbertson, Scott, and Bennett (1972). The 61 data sets for February 3, 1965, to May 4, 1966, for the Rio Grande reach which are used in the current study were obtained directly from the report by Culbertson, Scott, and Bennett.

\section{COMPUTATION PROCEDURE}

Colby and Hembree (1955) and Hubbell and Matejka (1959) have presented explanations for the total-load computational procedure for the MEP. For review and because the RMEP uses the MEP computational procedure, the explanation by Hubbell and Matejka (1959) is repeated here; however, references are made only to the RMEP equations. A total-load computation is made using the RMEP and the data from plate 7 in the report by Hubbell and Matejka (1959).

The computation form (fig. 5) is separated into three major computation blocks, which are headed: block I, preliminary data and computations; block II, computation of $i_{B} Q_{B}$; and, block III, computation by Z.

Step 1.-Fill in line 1, block I, with the basic data in pound-footsecond units; $w$, width; $V$, mean velocity; $d$, mean depth; $d_{s}$, average depth at the verticals sampled; $D_{65}$ and $D_{35}$, particle size at which 65 and 35 percent, respectively, of the bed material by weight is finer; Conc, measured suspended-sediment concentration (in parts per million); $Q_{s . M}$, measured suspended-sediment discharge (in tons per day); and Temp, water temperature in degrees Fahrenheit. (Except when noted otherwise, the English system of units is used throughout the computation.)

Step 2.-Solve equations 41 and 42 , line 2 , block 1 , for $(R S)_{R M}$ after assuming a trial $x$ where $\sqrt{(R S)_{R M}}$ equals $\left(V_{*}\right)_{R M} / \sqrt{8 ; x}$ usually is about 1.0 .

Step 3.-Compute $(R S)_{R M}$ from the value for $\sqrt{(R S)_{R M}}$ determined in step 2.

Step 4.-Compute the shear velocity, $\left(V_{*}\right)_{R M}$, using the expression $\left(V_{*}\right)_{R M}=\sqrt{g(R S)_{R M}}$ in. which $\sqrt{(R S)_{R M}}$ is from step 2 and $g$ is the gravity constant, 32.2 feet per second. 


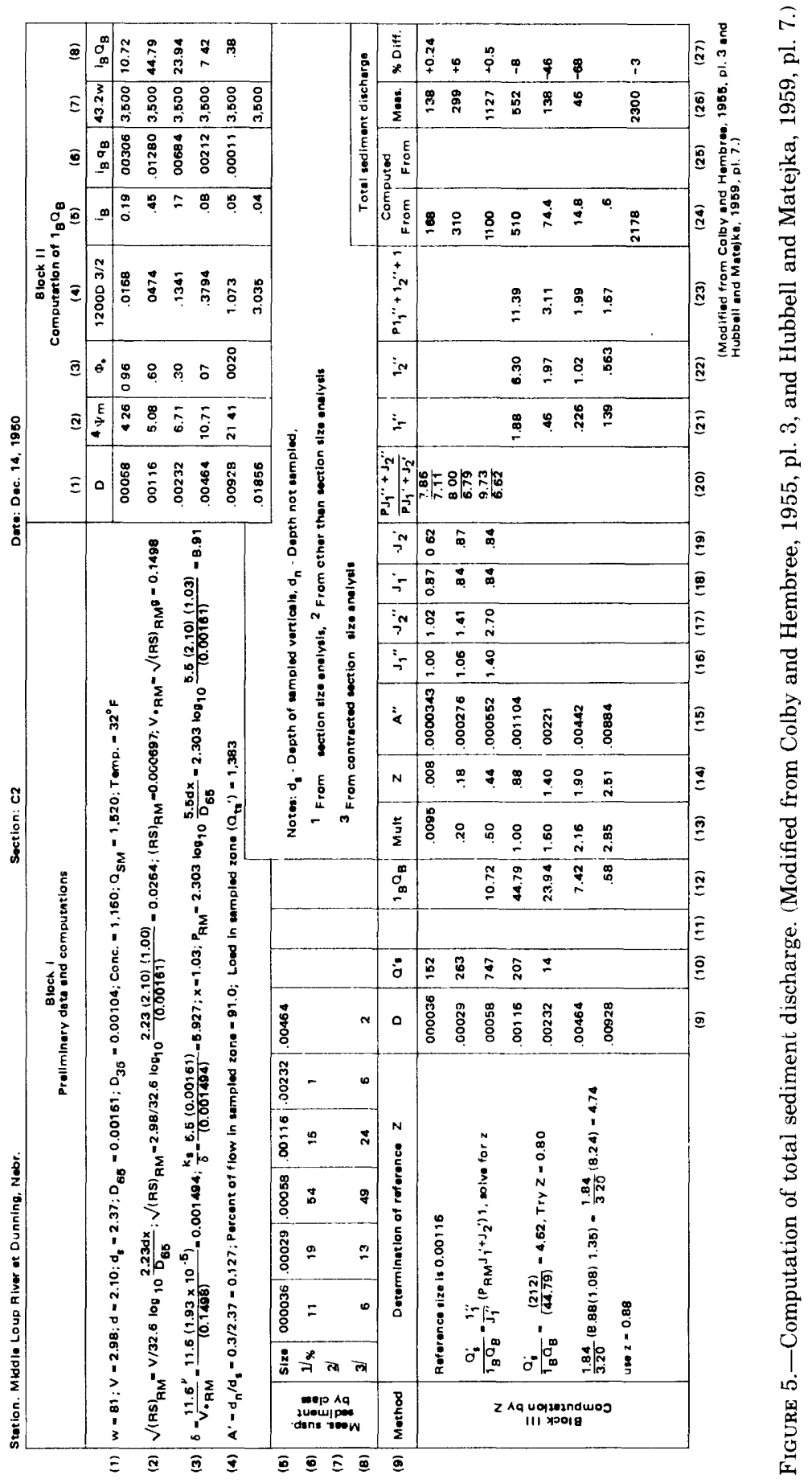


Step 5.-Compute $\delta_{R M}$ using the expression $\delta_{R M}=\frac{11.6 \mu}{\left(V_{*}\right)_{R M}}$ in which $\mu$ is the kinematic viscosity, in square feet per second, at the indicated water temperature and $\left(V_{*}\right)_{R M}$ is from step 4.

Step 6.-Calculate $\left(5.5 D_{65}\right) /(\delta)_{R M}$ and enter figure 4 for $x$. If the trial $x$ is not about the same as the $x$ from figure 4, repeat steps 2 through 6.

Step 7.-Solve for $P_{R . M}$ using equation $51-P_{R M}=2.303 \log \left(5.5 \frac{d x}{D_{65}}\right)$

Step 8.-Determine $A^{\prime}$ on line 4 from $A^{\prime}=d_{n} / d_{s}$, in which $d_{n}$ is the vertical distance not sampled, in feet; that is, the distance from the bottom of the sampled zone to the streambed.

Step 9.-From figure 6, find the percentage of flow in the sampled zone by using the computed $A^{\prime}$ and $P_{R M}$.

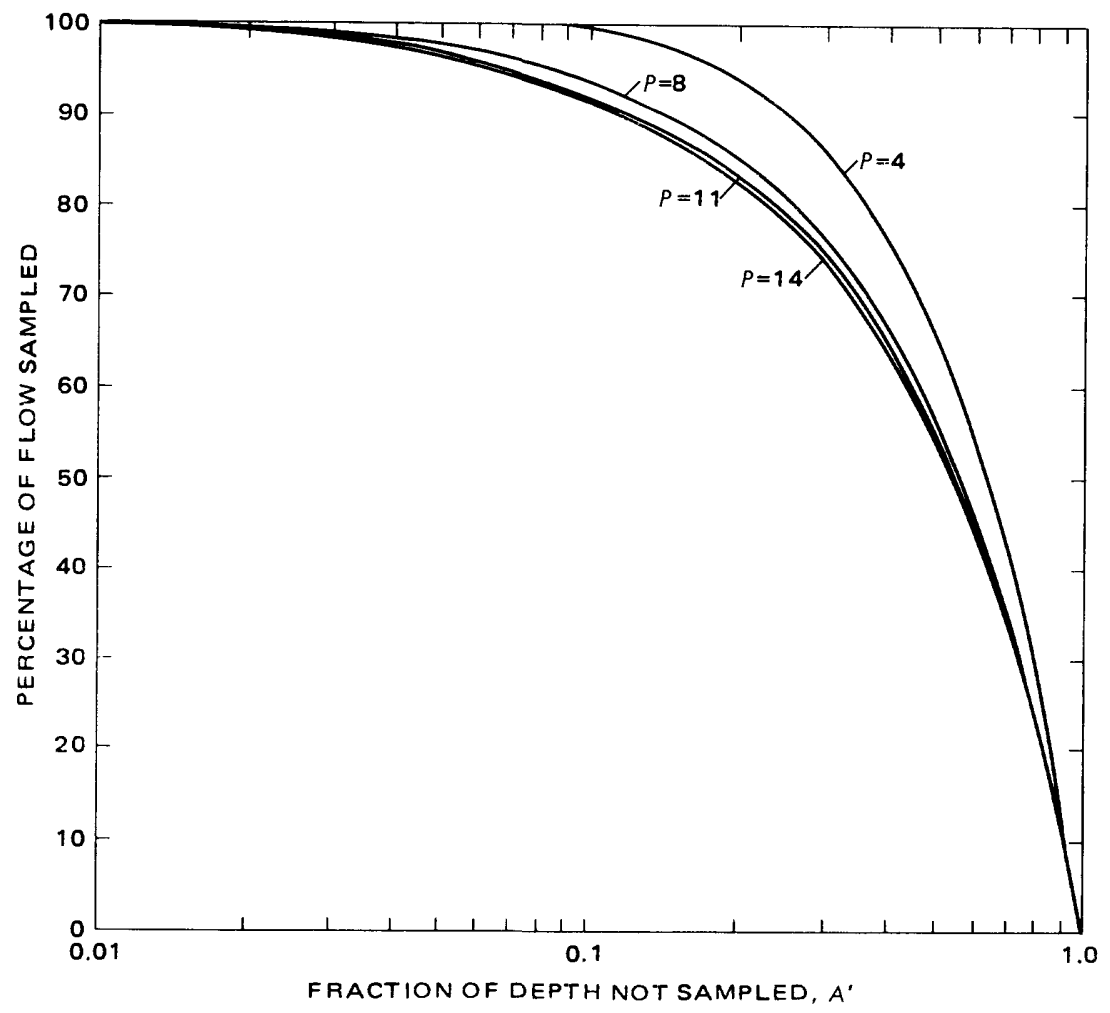

Figure 6.-Vertical distribution of streamflow. (From Colby and Hembree, 1955, fig. 45.) 
Step 10.-Compute the load in the sampled zone $\left(Q_{t s}{ }^{\prime}\right)$ by multiplying the percentage of flow in the sampled zone by the measured suspended-sediment discharge $\left(Q_{s m}\right)$.

Step 11.-List the percentage of measured suspended sediment in each size range under the geometric mean size of the range (line 5) on line 6 if the percentages were determined from an actual size analysis or on line 7 if they were determined by some other means. Line 8 is not completed unless the size distribution of the measured total load is known and is to be used in checking the accuracy of the computed total load.

Step 12.-List in column 1, block II, each geometric mean size of the size ranges that have appreciable bedload discharge $\left(i_{R} Q_{R}\right)$.

Step 13.-Compute a value for $(\psi *)_{R M}$ for each size range using the following expressions:

where

$$
\begin{gathered}
\left(\psi_{*}\right)_{R M}=1.65\left(\beta / \beta_{X_{1}}\right)^{2}\left(\delta_{R M}+C_{s} D\right) /(R S)_{R M} \text { or } \\
\left.\left(\psi_{*}\right)_{R M}=1.65\left(\beta / \beta_{x_{1}}\right)^{2}(D) / R S\right)_{R M} \text { if } D>\left(\delta_{R M}+C_{s} D\right)
\end{gathered}
$$

$$
\begin{aligned}
\beta= & \log (10.6) \\
\beta_{x_{1}}= & \log (10.6 x) \\
C_{s}= & 0 \text { if }\left[\left(D_{65}\right)\left(D_{35}\right)\right]_{1 / 2}, \text { in millimeters, is less than } 0.20 ; \text { and } \\
C_{s}= & 1.00 \text { if }\left[\left(D_{65}\right)\left(D_{35}\right)\right]_{1 / 2,}, \text { in millimeters, is larger than } 0.52 ; \\
& \text { otherwise }
\end{aligned}
$$

$$
C_{s}=-0.62+3.12\left[\left(D_{65}\right)\left(D_{35}\right)\right]^{1 / 2}
$$

in which $D_{65}$ and $D_{35}$ are in millimeters.

Equation 49 is based entirely on the data sets for sites on the Niobrara and the Middle Loup Rivers. The following steps were taken in developing the equation:

1. Using different numerical values for $C_{s}$ in equation 49 , total sediment discharges were computed for each set of data for the Niobrara and Middle Loup sites.

2. The difference between computed and measured total sediment discharge were determined for each data set, and the square root of the mean variance for the Niobrara and Middle Loup data were computed.

3. Graphs showing the relation between the value of $C_{s}$ used in step 1 and the square root of the mean variance obtained in step 2 were developed.

4. The values of $C_{s}$ for the minimum square root of the mean variance were determined from the relation developed in step 3; a value of 0.25 were determined for the Niobrara data and a value of 0.50 were determined for the Middle Loup data. 
5. Averages of the geometric means of $D_{35}$ and $D_{65}$, in millimeters, were determined for the Niobrara data and for the Middle Loup data; these averages were 0.28 and 0.36 , respectively.

6. The values from step 3 and step 5 were plotted on a graph and a straight line was drawn through the two points; this line is represented by equation 49 .

Step 14.-Determine $\phi_{*}$ for each size range from a curve in Einstein (1950, fig. 10) using the $\left(\psi_{*}\right)_{R M}$ from step 13.

Step 15.-List the value of $1,200 D^{3 / 2}$ for each size range in column 4. $D$ is the geometric mean size of the size range.

Step 16.-Enter the fraction of bed material in each size range $\left(i_{b}\right)$ in column 5 .

Step 17.-Compute $i_{B} q_{B}$ for each size range using the expression $i_{B} q_{B}=1,200 i_{b} D^{3 / 2} \phi_{*}$.

Step 18.-Compute the constant $43.2 w$ for entry in column 7 . The width, $w$, is the effective width-width determined by applying an appropriate horizontal angle correction to the width rather than the velocity or discharge, and 43.2 is a constant converting pounds per second to tons per day.

Step 19.-Compute $i_{B} Q_{B}$ for each size range and enter in column 8; $i_{B} Q_{B}$ is the product of $i_{B} q_{B}$ and $43.2 w$.

Step 20.-List in column 9, block III, the geometric mean size of all the size ranges that are transported. (Column numbers for block III are at the bottom of the table.)

Step 21.-Compute the load in the sampled zone of each size range $\left(Q_{s}{ }^{\prime}\right)$ as the product of the load in the sampled zone $\left(Q_{t s}{ }^{\prime}\right)$ (line 4 , block I) and the percentage of measured suspended sediment in each size range (from line 6, block I). Enter the product in column 10.

Step 22.-Copy in column 12 each $i_{B} Q_{B}$ from column 8, block II.

Step 23.-Compute $A^{\prime \prime}$ for each size range from $A^{\prime \prime}=2 D / d$ and enter in column $15 . A^{\prime \prime}$ is equal to the distance of the lower limit of integration above the stream bed, $a$, divided by $d$. Einstein (1950, p. 25) shows the relations, $a=2 D$.

Step 24.-The large box headed "Determination of Reference $z$," in block III is used in this step. Select the reference size. The reference size should be the geometric mean size of a size range that is represented in appreciable quantities in both bedload discharge and suspended-sediment discharge. For streams in which the bedload discharge is a substantial part of the total load, the reference size range ordinarily will be the range that has the greatest total sediment discharge. Once the reference size is selected, it can be used for all subsequent computations. Compute the value of the ratio $Q^{\prime}{ }_{s}^{\prime} / i_{B} Q_{B}$ (the left-hand side of the formula $Q^{\prime}{ }_{s} i_{B} Q_{B}=\frac{I_{1}^{\prime \prime}}{J}\left(P_{R . N} J_{1}{ }^{\prime}+J_{2}^{\prime}{ }_{2}\right)$ ) for the 
reference size. Assume a $z$ for the reference size (figure 7 will aid in a first approximation for many shallow streams) and solve the righthand side of the above formula with values determined by using $A^{\prime}$ and $A^{\prime \prime}$ and the assumed $z$ with figures 5, 6, and 7 of Colby and Hembree (1955) or figures 9, 10, and 13 of Hubbell and Matejka (1959). When the right and left sides of the equation balance, the assumed $z$ is the proper $z$ for the reference size range.

Step 25.-Because $z$ varies with about the 0.7 power of fall velocity and because flow characteristics that might cause $z$ to vary are constant at a given cross section and time, the $z$ for the other size ranges can be computed by using equation 40 and $z$ for the reference size range. Curves for the different size ranges of $\left(V_{s u} / V_{s r}\right)^{0.7}$ (called multipliers) versus temperature were plotted by Colby and Hembree (1955, pl. 1); the 0.25 to 0.50 millimeter size range is the reference range. The $z$ for any size range is the product of the $z$ for the reference range and the multiplier at the indicated water temperature for the size range. List in column 13 the multiplier with each size range.

Step 26.-Compute the $z$ for all size ranges from the $z$ for the reference range and the multiplier.

Step 27.-Complete columns 16, 17, 18, and 19 for the ranges for sediment sizes smaller than size in the reference size range; use curves in Colby and Hembree (1955, figs. 5 and 6) and the $z, A^{\prime}$, and $A^{\prime \prime}$ for each size range.

Step 28. - Compute the ratio $\left(P_{R M 1} J_{1}^{\prime \prime}+J_{2}{ }^{\prime \prime}\right) /\left(P_{R .1} J_{1}{ }^{\prime}+J_{2}{ }^{\prime}\right)$. List the ratios in column 20 .

Step 29.-Complete column 21 and 22 for the ranges of sediment sizes not included in Step 27; use curves in Colby and Hembree (1955, figs. 4 and 7) and the $z$ and $A^{\prime \prime}$ for each size range.

Step 30.-Compute $P_{R M} I_{1}{ }^{\prime \prime}+I_{2}{ }^{\prime \prime}+1$ for entry in column 23 using the information in columns 21 and $22 . I_{2}{ }^{\prime \prime}$ is always negative.

Step 31.-Compute the total sediment discharge for the ranges for sediment sizes smaller than the size in the reference size range; it is the product of the values in column 20 and in column 10. Enter the total sediment discharge in column 24 .

Step 32.-Compute the total sediment discharge of all the size ranges not included in step 31 ; it is the product of the values in columns 12 and 23. Enter the total sediment discharge in column 24.

Computer programs analogous to the MEP and RMEP were used to compute sediment discharge. Documentation for the computer program for MEP, called MODEIN, is available in a report by Burkham, Kroll, and Porterfield (1977). The computer program for the RMEP, called RMODEIN, is the same as MODEIN except that pertinent equations have been changed. 


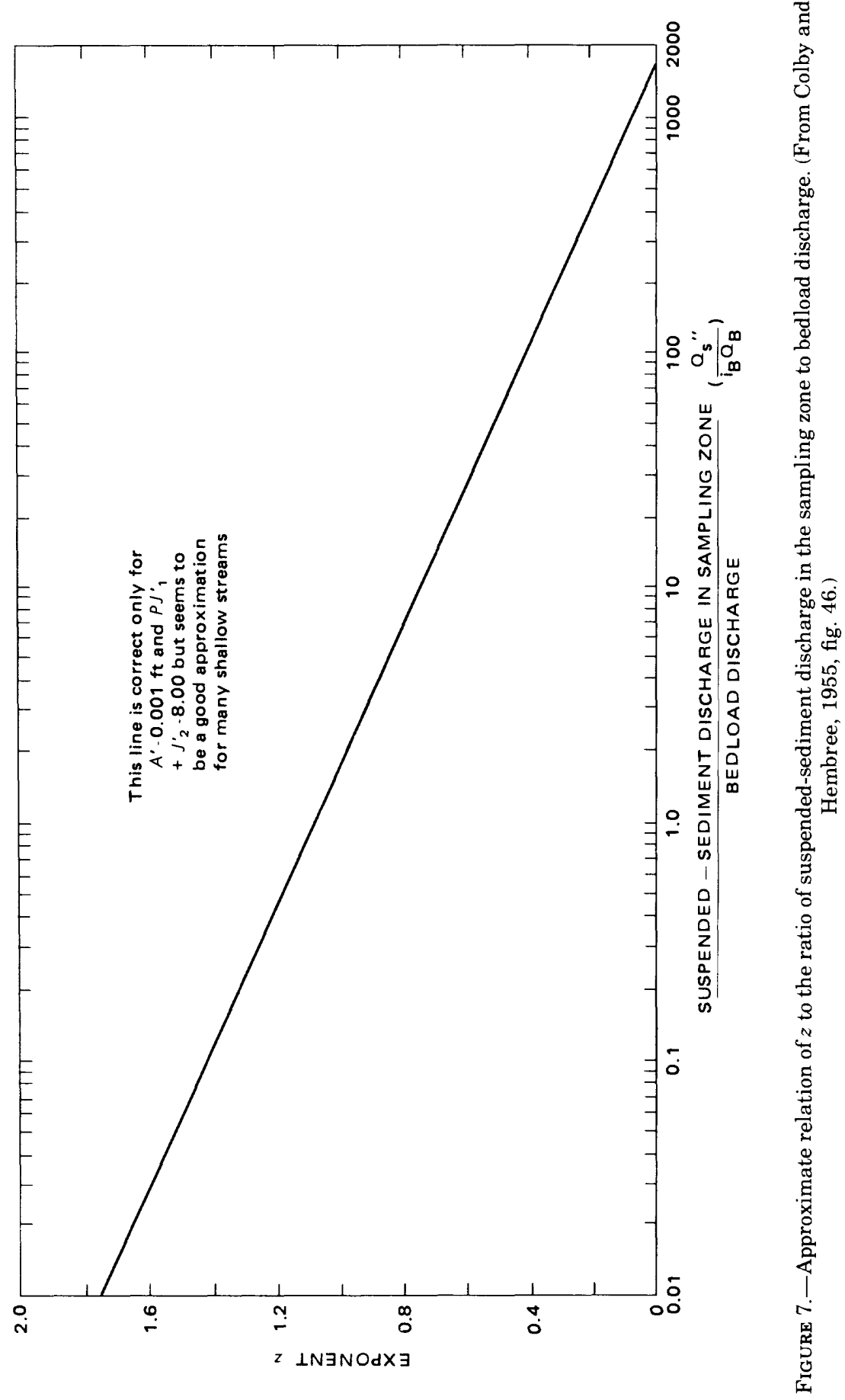




\section{RESULTS OF COMPARISON}

\section{INTRODUCTION}

Sediment discharges, by size ranges and for the total, computed by the MEP and RMEP for each data set are compared with measured values. The following statistics were computed for the comparisons: the percentage of the total measured sediment discharge for each sediment-discharge computation; the average and standard deviation of these percentages for each of the five data groups; the square root of the mean variance for the computed values for the size ranges and for the total sediment discharge; mean of measured and computed sediment discharges for the size ranges and for the total sediment discharge; and ratio of square root of mean variance to mean of measured sediment discharges for the size ranges and for the total sediment discharge. The percentage of the total measured sediment discharge is the ratio of computed total sediment discharge to measured total sediment discharge multiplied by 100 . The square root of the mean variance which was used as an approximation of the standard error was determined using the expression

in which

$$
S E_{Q}=\sqrt{\sum_{i=1}^{N_{s}}\left(Q_{T M_{i}}-Q_{T c_{i}}\right)^{2} /\left(N_{s}-1\right)} ;
$$

$S E_{Q}=$ the square root of the mean variance;

$Q_{T M}=$ the measured sediment discharge;

$Q_{T C}=$ the computed sediment discharge; and

$N_{s}=$ the number of measurements in the set.

The measured-sediment data may contain significant errors; in which case, the standard error of estimate, $S E$, would be different than $S E_{Q}$. The ratio of square root of mean variance to mean of measured sediment is used, when multiplied by 100 , as a rough approximation of the standard error of estimate in percent.

\section{NIOBRARA RIVER}

The RMEP apparently gave better results than the MEP for the Niobrara site (table 1). This premise is based on the following facts and criteria:

1. For the total sediment discharge, the square root of the mean variance for the 21 data sets is 415 tons per day for the RMEP and 674 tons per day for the MEP.

2. The mean of the computed total sediment discharge determined according to the RMEP is more nearly equal to the measured mean 
than that for computed totals determined according to the MEP. The percentage of measured sediment ranged from 55 to 137 and averaged 100 percent for the RMEP; the range was 70 to 148 and the average was 94 for the MEP.

3 . Using the average of the measured sediment discharges, 2,310 tons per day, as a gage, the approximate standard error of estimate is about 18 percent for the RMEP and about 29 percent for the MEP.

4. For four of the five ranges of particle sizes, the approximate standard error of estimate for results obtained according to the RMEP apparently was better than that for the MEP. For most of the data sets for the other range, only a relatively small amount of sediment was being transported.

For the Niobrara site, the RMEP and the MEP give better results for the ranges of relatively small particles than for the ranges of relatively large particles. This should be expected because the computation for the small particles is based primarily on measured data while that for the large particles is based largely on theoretical considerations.

Statistics different from those in table 1 were reported in a previous paper (Burkham and Dawdy, 1976b). The previous paper was primarily a progress report and the statistics were of a preliminary nature.

\section{MIDDLE LOUP RIVER}

The RMEP and the MEP gave results for the site on the Middle Loup River that have about the same accuracy (table 1). The square root of the mean variance for the computed total sediment discharge is 298 tons per day for the RMEP and 310 tons per day for the MEP. Using the average of the measured total sediment discharges, 1,570 tons per day, as a gage, the approximate standard error of estimate is about 20 percent for both methods. The percentage of measured total sediment discharge ranged from 65 to 146 and average 105 for the RMEP; the range is 68 to 165 and the average is 106 for the MEP. As for the Niobrara data, both methods gave better results for ranges for relatively small particles than for ranges for relatively large particles.

Nine of the fifty-nine data sets were not used in determining the statistics for variance and ratios for the $1.00-$ to $2.00-\mathrm{mm}$ range. This was done to minimize the possibility of introducing bias in estimating errors for the two methods. For the nine data sets, the measured or computed value of sediment discharge is 0 . For unlimited sets of data containing 0 , the mean of the deviations of computed values from measured values could not be 0 , a requirement in determining an unbiased estimate of errors. 
FIVEMILE CREEK AT SHOSHONI

The RMEP and the MEP yielded computed values for total sediment discharge for the Shoshoni site that have about the same approximate standard error of estimate (table 1). For the RMEP, the approximate standard error of estimate for total sediment discharge is 383 tons per day; it is 391 tons per day for the MEP. Using the average of the measured total sediment discharges, 1,730 tons per day, as a gage, the approximate standard error of estimate for totalsediment discharge is about 23 percent for both procedures.

Both procedures gave values for total sediment discharge that are significantly larger than measured values (table 1). The average of the percentage of measured sediment discharge is 115 for the RMEP and 122 for the MEP. The ratio of the average of computed total sediment discharge to the average of measured total sediment discharge is about 1.15 for both procedures.

The reason that the computed total sediment discharge is larger than the measured value is now known, however, it could result from either the total sediment discharge at the weir being significantly undermeasured, or the total sediment discharge being overcomputed, or both.

The data-set sample for the different sediment-size ranges for the Shoshoni site is relatively small, therefore, statistics for the sample probably are not meaningful. The different statistics, however, were computed (table 1).

\section{FIVEMILE CREEK AT RIVERTON}

The RMEP and the MEP gave computed values for total-sediment discharge for the Riverton site that have about the same approximate standard error of estimate (table 1). The approximate standard error of estimate for total sediment discharge is 249 tons per day for the RMEP and 228 tons per day for the MEP. Using the average of the measured total sediment discharges, 1,360 tons per day, as a gage, the approximate standard error of estimate for total sediment discharge is about 18 percent for both procedures.

Again, both procedures give values for total sediment discharge that are significantly larger than measured values. The average of the percentages of measured-sediment discharges is 107 for the RMEP and 112 for the MEP. The ratio of the average of computed total sediment discharge to the average of measured-total-sediment discharge is 1.11 for both procedures.

The statistics for the sediment-size ranges which are given in table 1 probably are not meaningful because the data-set sample is relatively small. 
RIO GRANDE CONVEYANCE CHANNEL

The approximate standard error of estimate for total sediment discharge for the Rio Grande data is 2,510 tons per day for both procedures (table 1; fig. 8). Using the average of the measured total sediment discharge, 8,860 tons per day, as a gage, the approximate standard error of estimate for total sediment discharge is 28 percent for both procedures.

Both procedures gave average values for total sediment discharge that are significantly smaller than the average of measured values; the average of percentages is 86 for both procedures. The relatively low averages of computed values apparently result because the amount of sediment in the 0.062 to $0.125 \mathrm{~mm}, 0.125$ to $0.250 \mathrm{~mm}$ and 0.250 to $0.500 \mathrm{~mm}$ size ranges is underestimated during periods when the bed form is flat or in transition (fig. $8 \mathrm{~A}, \mathrm{C}$ ). Conversely, for these size ranges, the amount of sediment being moved during periods when the bed form is dunes is overestimated (fig. $8 B$ ).

Earlier it was noted that Einstein made the assumption that the energy of turbulence generated by form resistance does not contribute significantly to bed-material motion. These data tend to support that assumption and, in fact, tend to indicate that the form resistance actually may inhibit bedload transport. Perhaps a portion of the bed, that immediately downstream from the dunes which is influenced by upstream eddies, does not contribute to downstream motion. That area could easily comprise 10 percent of the bed and fully account for the underestimation evidenced for dune conditions.

The RMEP can be readily adjusted by applying empirical corrections so that it will give results that agree better with measured sediment discharges for the Rio Grande data. For example, the indicated better agreement shown in figure $8 D$ was obtained by using equation $46 \mathrm{~A}$ to represent $\psi_{* R M}$ for all data sets for the Rio Grande site for May 4, 1966. Equation 46A is

$$
\psi_{* R M}=0.50\left(\beta / \beta_{x 1}\right)^{2} D /(R S)_{R M}
$$

which was obtained by applying a correction, $C_{6}=0.30$, to equation 46. Other than to force an agreement, a rational reason for applying the correction, $C_{6}$, is not apparent at this time. Because of this and because data for which bed form is described are not adequate for testing when an empirical correction is applied, other equations to force agreement between computed and measured-sediment discharge for the different bed forms were not developed. Equation 46A probably should not be used because it has not been tested.

A more fundamental approach to adjust RMEP would be to determine more exactly the proper representative particle size that should 

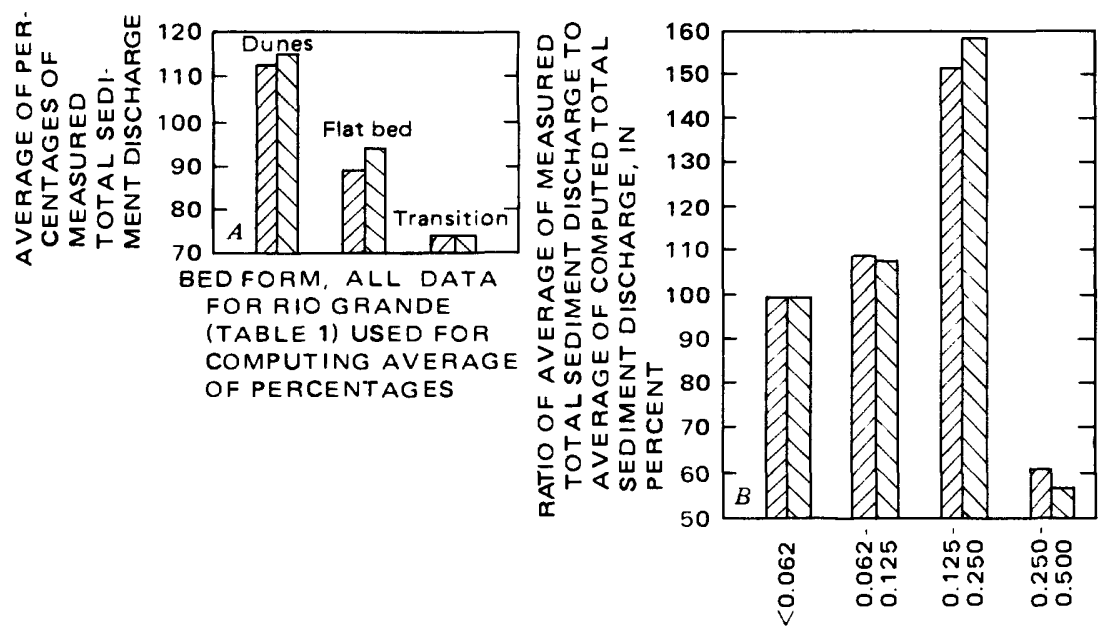

SIZE RANGES, IN MILLIMETERS, FOR DUNES ALL DATA FOR RIOGRANDE SITE USED FOR COMPUTATIONS
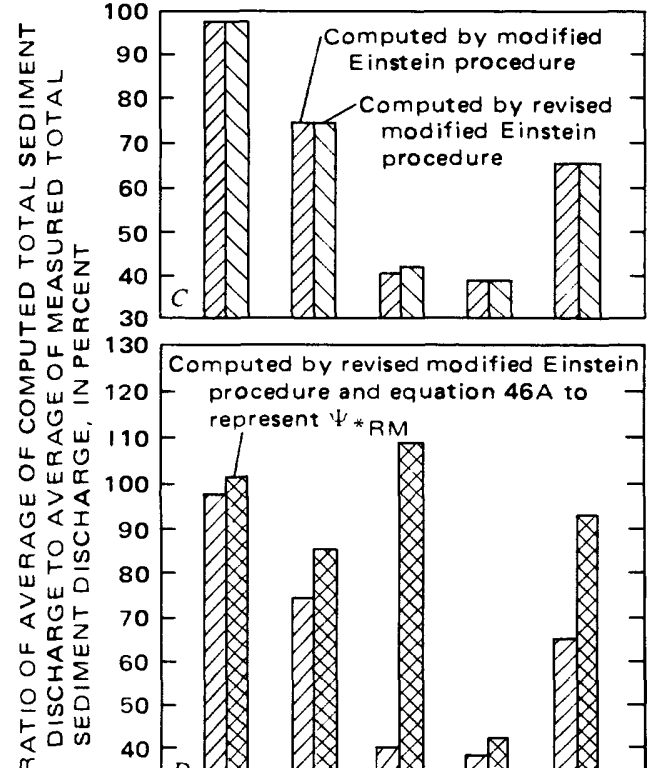

5 出 130

$\sum_{w} \underline{z}$

Computed by revised modified Einstein

0

$\propto 0$

$\omega>\varangle$

$4<\frac{1}{0}$

$\varangle$ 에

${ }_{\omega}^{\infty} \overline{0}$

405

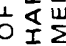

으늠

\&㟔䋆 120

procedure and equation $46 \mathrm{~A}$ to

$110-$ represent $\Psi^{*} R M$
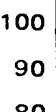

80

70
60

60
50
40

30 
be used to represent $k_{s}$. The parameter $5.5 D_{65}$ is an average value based on a best fit for several sites (Burkham and Dawdy, 1976a). Certainly $k_{s}>D_{65}$, and on the average it is on the order of $5.5 D_{65}$. A future study which better determines $k_{s}$ and allows the multiplier, $x_{0}$, to be related to the size distribution may further improve the application of the RMEP.

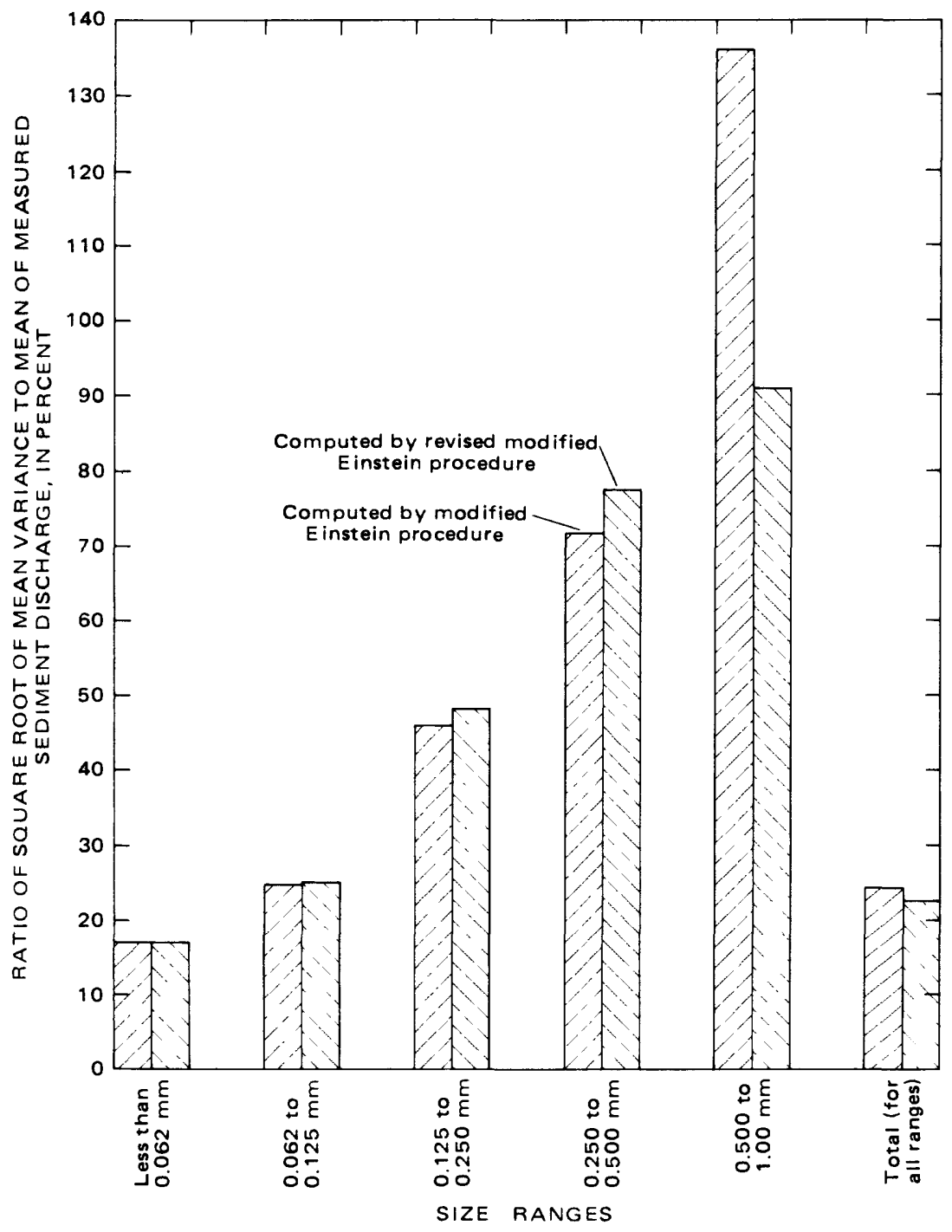

FigURE 9.-Weighted approximate standard error of estimate for the different ranges of particle sizes and for the total sediment discharge for five data groups. 


\section{CHARACTERISTICS FOR THE FIVE DATA GROUPS}

The weighted approximate standard error of estimate for the different ranges of particle sizes is given in figure 9 . The weighted percentage for a range size was obtained using the equation

$$
S E_{u}=\sqrt{\frac{\sum\left[\left(S E_{1}\right)^{2}\left(n_{1}\right)+\ldots\left(S E_{5}\right)^{2}\left(n_{5}\right)\right]}{\sum\left(n_{1}+\ldots n_{5}\right)}}
$$

in which $S E_{1} \ldots S E_{5}$ denotes the approximate standard error for the particle-size range for the five data groups and $n_{1} \ldots n_{5}$ represents the number of data sets for the particle-size range and data group. The Rio Grande data for the range for the 0.500 to $1.00 \mathrm{~mm}$ particle sizes were not used in computing a weighted approximate standard error of estimate; the quantity of sediment being transported for the range was small.

Figure 9 shows the weighted percentage error by size class for the total load of sands transported for the five data groups. For the smaller sizes, most of the sediment is moving in suspension, therefore large errors of estimation of that portion moving as bedload still result in small errors in terms of percent of total load for a class. For large sizes, almost all the sediment is moving as bedload, and any error in estimation of the bedload is translated almost directly into errors in total load.

The data for standard errors for the range of particle sizes less than $0.062 \mathrm{~mm}$ offers an excellent opportunity to approximate the standard sampling error for wash-load discharge for the diversified flow and sediment conditions found at the five study sites. A wash-load sampling error is the error introduced in determining the wash-load discharge by sampling. The wash-load sampling error depends on the accuracy of the water-discharge measurement, the sedimentsampling instrument, the sediment-sampling technique-including sampling time and the fluctuation in sediment concentration during this time-laboratory analysis, and the computation of concentration. In order to approximate the standard sampling error, the following assumptions were necessary:

1. The sampling error for wash load for a normal section is independent of the sampling error for wash load for a weir or contracted section.

2. The mean sampling error for wash load for a section is zero.

3. For comparable periods of records and discharges for a site, the true wash load at a normal section equals the true wash load at a weir.

4. For a site, the standard wash-load sampling error for a normal section equals the standard wash-load sampling error for a weir. 
Considering these assumptions, the approximate standard error of 17 percent (the 17 percent is the weighted approximate standard error of estimate that is shown in figure 9 for the range of particle sizes less than $0.062 \mathrm{~mm}$ in diameter) is significantly larger than the standard error for wash load. The variances for independent errors are additive, therefore, the washload sampling error can be represented as:

$$
\sqrt{2(S E)^{2}{ }_{u}}=17 \text { or }(S E)_{w}=12.0 \text { percent }
$$

The 12.0 percent represents the standard wash-load sampling error for the five study sites.

The 12.0 percent for particle sizes less than $0.062 \mathrm{~mm}$ probably is a better approximation of the true standard error of estimate for the RMEP and MEP than the 17 percent that is shown in figure 9. The values shown in figure 9 for the other ranges of particle sizes also probably are slightly biased on the high side because they are computed as a difference of two terms. The amount of bias, however, cannot be determined because the relative sizes of the two components of error cannot be estimated as they were for the wash load.

Based on figure 10, the ratio of computed to measured total sediment discharge apparently increases with an increase in the median diameter of sediment particles in a mixture. Figure 10, however, may be misleading. As previously discussed for the Rio Grande site, the total sediment discharge is underestimated for periods when the bed form was flat or in transition and overestimated for periods when the bed form was dunes. A sediment bed in motion in a normal section usually does not retain a fixed bed form for all flow conditions, therefore, the bed form at the sites on the Niobrara, Middle Loup, and Fivemile Rivers probably is dunes, flat, or in transition at different times. If so, the results found for the Rio Grande site-overestimates for dunes and underestimates for flat bed-probably applies for the other sites. The fact that the total sediment discharge is overestimated for a site may only indicate that a bed form of dunes was at the normal section during most of the periods when sediment transport data were being obtained.

\section{SUMMARY AND CONCLUSIONS}

The modified Einstein procedure (Colby and Hembree, 1955) is revised by applying corrections to equations 29 and 39 and by replacing equations 27 and 28 with equations 46 and 48 . The revision was made because $D_{65}$ was used directly in the modified Einstein procedure to represent the equivalent sand roughness, $k_{s}$, whereas a much larger size apparently should have been used (Burkham and Dawdy, 1976a). For this study, $5.5 D_{65}$ an average value based on a best fit for data for 


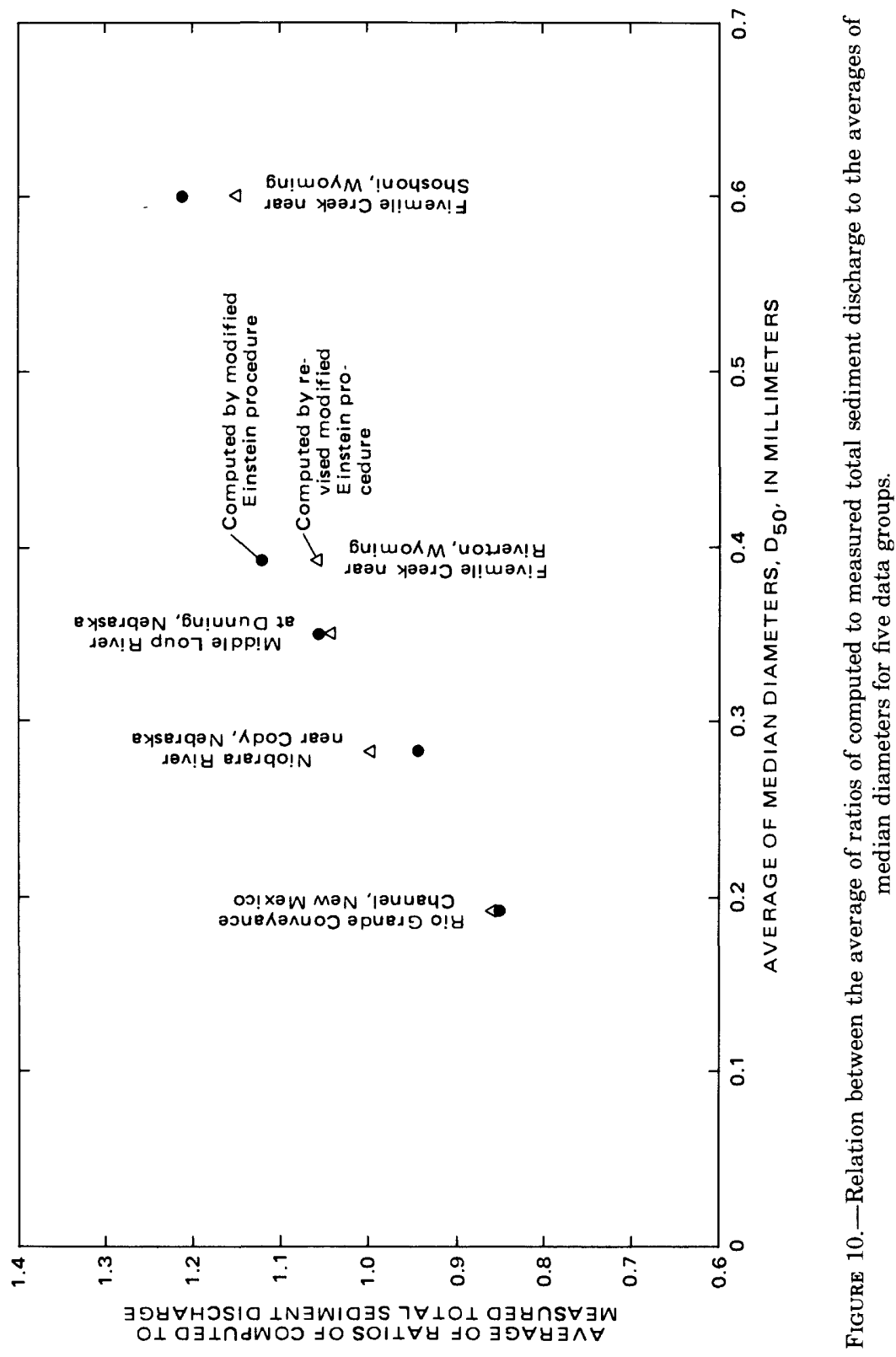


several sites, was used. The modified and the revised modified Einstein procedures were tested by comparing computed and measured sediment discharge for 292 sets of data for five sites in sand-channel streams.

Conclusions resulting from the study are:

1. The revised modified Einstein procedure apparently is an improvement over the modified Einstein procedure. For the sediment and flow conditions tested, the computed sediment discharges apparently are not significantly more accurate; however, improvement is indicated because the correction of pertinent equations eliminates some of the empirical adjustments contained in the modified Einstein procedure.

2. The revised procedure eliminated the need to arbitrarily divide the bedload transport intensity, $\phi_{*}$, by 2 as was done in the modified Einstein procedure (Colby and Hembree, 1955). Values for $\phi_{*}$ are taken from a curve relating $\psi_{*}$ intensity of transport, and $\phi_{*}$. The $\psi_{*}$-to- $\phi_{*}$ curve is theoretical; however, it was verified by experimental data (Einstein, 1950). Einstein's equation for bedload (Einstein, 1950 , p. 59, step 34) which was used in the modified and revised modified Einstein procedures requires that values taken from the $\psi_{*}$-to- $\phi_{*}$ curve be used directly to represent the bedload transport intensity.

3. For the same data, the revised modified Einstein procedure gives shear velocities that are significantly larger and $x$ values that are significantly smaller than the corresponding values computed using the modified Einstein procedure. The ratio of shear velocity for the revised modified Einstein procedure to the shear velocity for the modified Einstein procedure ranges from 9.52 to 1.28 for stream velocities ranging from 0.50 to 15 feet per second. For the 292 data sets used in testing the two procedures, the value of $x$ ranged from 1.00 to 1.14 for the revised modified Einstein procedure and from 1.00 to 1.62 , the maximum value possible, for the modified Einstein procedure. The value of $x$ is 1 for fully turbulent flow.

4. Based on data sets for the five sites, the approximate standard error of estimate for total sediment discharge determined by the two procedures is about 22 percent. Except for the range for the largest particles tested, the weighted standard error is approximately equal between the two methods for the different sediment-size ranges. Weighted standard errors for the different sediment-size ranges for the MEP and RMEP, respectively, are: 17 percent and 17 percent for sizes smaller than $0.062 \mathrm{~mm} ; 25$ percent and 25 percent for sizes from 0.062 to $0.125 \mathrm{~mm} ; 46$ percent and 48 percent for sizes from 0.125 to $0.250 \mathrm{~mm} ; 72$ percent and 77 percent for sizes 0.250 to $0.500 \mathrm{~mm}$; and 136 percent and 91 percent for sizes 0.500 to $1.00 \mathrm{~mm}$. The standard 
error for wash load is relatively low because the wash load is determined directly by sampling and the accuracy of values determined by sampling is better than those determined indirectly by equations, which is done for relatively large particles moving as bedload. The approximate standard error for total sediment discharge is only about 22 percent because a large fraction of the total sediment discharge was in suspension and the suspended-sediment discharge is determined directly by sampling.

5. For a site on the Rio Grande conveyance channel, the amount of sediment in the 0.062 to $0.125 \mathrm{~mm}, 0.125$ to $0.250 \mathrm{~mm}$, and 0.250 to $0.500 \mathrm{~mm}$ sizes ranges were significantly underestimated by the two procedures for periods when the bed form was flat or was in transition. Conversely, for these ranges of sediment sizes and for the total, the amount of sediment being moved was significantly overestimated for periods when the bed form was dunes.

6. For two sites on Fivemile Creek in Wyoming, the total sediment discharge is significantly overestimated. This statistic may be misleading. A sediment bed in motion at a normal section usually does not retain a fixed bed form for all flow conditions, therefore, the bed form at the sites on Fivemile River probably is dunes, flat, or in transition at different times. If so, the results found for the Rio Grande site probably apply for the other sites (see conclusion 5). The fact that the total sediment discharge is overestimated for a site may only indicate that a dune bed form was at the normal section during most of the periods when sediment transport data were being obtained. Information on the type of bedform at the time samples were taken was not available.

7. The revised modified Einstein procedure needs further study before it is considered as a replacement for the modified Einstein procedure. The revised procedure has been tested for a wider range of flow and sediment conditions than those for which the modified Einstein procedure was originally tested. Basically, however, the revised procedure still has only been tested for sand-size sediments. Tests for conditions in which large amounts of coarse sediment-gravel and small boulders-are moved as bedload have not been made; these tests will be feasible only after good data (usable as measured values) for the bedload movement of coarse sediment are available.

8. Given more test data, a future study which determines more exactly the proper representative particle size to be used to represent $k_{s}$ might be fruitful. The study should allow the multiplier $x_{0}$ to be related to the size distribution as well as the percentile fractile used to represent roughness height; the multiplier $x_{0}$ probably should gradually decrease from some positive value to 1 as the size distribu- 
tion approaches uniformity and as the percentile fractile used to represent roughness height approaches 100 .

9. The revised modified Einstein method, like the modified Einstein method, requires much experience and judgment in order to obtain reliable results.

10. New and innovative approaches for explaining the physical principles of the bed-form phenomena as related to sediment transport are needed in order that the method presented by Colby and Hembree - the modified Einstein method - can be moved towards the original intent of the Einstein procedure, an approach based on physical principles.

\section{REFERENCES CITED}

American Society of Civil Engineers, 1975, Sedimentation engineering: New York, 745 p.

Barnes, H. H., 1967, Roughness characteristics of natural streams: U.S. Geological Survey Water-Supply Paper 1849, 213 p.

Burkham, D. E., and Dawdy, D. R., 1976a, Resistance equation for alluvial channel flow: Journal of the Hydraulic Division, Proceedings of the American Socity of Civil Engineers, v. 102, no. HY 10, p. 1479-1489.

1976b, Proposed revision to the Modified Einstein Method of computing total sediment discharge, in Proceedings of the Third Federal Inter-Agency Sedimentation Conference, Sedimentation Committee, Water Resources Council, p. 437-449.

Burkham, D. E., Kroll, C. G., and Proterfield, George, 1977, A guide for the application of the computer program for the modified Einstein method of computing total sediment discharge (MODEIN): U.S. Geological Survey Computer Contribution, NTIS series, $138 \mathrm{p}$.

Colby, B. R., 1961, Discharge of sand and mean-velocity relationships in sand-bed streams; U.S. Geological Survey Professional Paper 462-A, 47 p.

Colby, B. R., and Hembree, C. H., 1955, Computation of total sediment discharge Niobrara River near Cody, Nebraska: U.S. Geological Survey Water-Supply Paper $1357,187 \mathrm{p}$.

Colby, B. R., Hembree, C. H., and Rainwater, F. H., 1956, Sedimentation and chemical quality of surface waters in the Wind River basin, Wyoming: U.S. Geological Survey Water-Supply Paper 1373, 336 p.

Culbertson, J. K., and Dawdy, D. R., 1964, A study of fluvial characteristics and hydraulic variables, Middle Rio Grande, New Mexico: U.S. Geological Survey Water-Supply Paper 1498-F, 74 p.

Culbertson, J. K., Scott, C. H., and Bennett, J. P., 1972, Summary of alluvial-channel data from Rio Grande conveyance channel, New Mexico, 1965-69: U.S. Geological Survey Professional Paper 562-J, 49 p.

Dawdy, D. R., 1961, Depth-discharge relations of alluvial streams-discontinuous rating curves: U.S. Geological Water-Supply Paper 1498-C, 16 p.

Einstein, H. A., 1950, The bed-load function for sediment transportation in open channel flows: U.S. Department of Agriculture Technical Bulletin 1026, 71 p.

Gonzales, D. D., Scott, C. H., and Culbertson, J. K., 1969, Stage-discharge characteristics of a weir in a sand-channel stream: U.S. Geological Survey Water-Supply Paper 1898-A, 29 p. 
Harris, D. D., and Richardson, E. V., 1964, Stream gaging control structure for Rio Grande conveyance channel near Bernardo, New Mexico: U.S. Geological Survey open-file report, $78 \mathrm{p}$.

Hubbell, D. W., Jordan, P. R., Culbertson, D. M., and Hembree, C. H., 1956, Progress report no. 1-Investigation of some sedimentation characteristics of a sand-bed stream: U.S. Geological Survey open-file report, $78 \mathrm{p}$.

Hubbell, D. W., and Matejka, D. Q., 1959, Investigations of sediment transportation Middle Loup River at Dunning, Nebraska: U.S. Geological Survey Water-Supply Paper 1476, $123 \mathrm{p}$.

Keulegan, G. H., 1938, Laws of turbulent flow in open channels: Research Paper 1151, U.S. National Bureau of Standards Journal of Research, v. 21 p. 701-741.

Leopold, L. B., Wolman, M. G., and Miller, J. P., 1964, Fluvial processes in geomorphology: San Francisco, W. H. Freeman and Co., 522 p.

Limerinos, J. T., 1970, Determination of the Manning coefficient from measured bed roughness in natural channels: U.S. Geological Survey Water-Supply Paper $1898-\mathrm{B}, 47 \mathrm{p}$.

Prandtl, Ludwig, 1926, Über die ausgelbildeter turbulenz (On fully developed turbulence): International Congress on Applied Mechanics, 2d, Zurich, 1926, Procedures, p. 62-74.

von Kármán, T., 1930, Mechanische aehnlichkeit und turbulenz (Mechanical similarity and turbulence): International Congress on Applied Mechanics, 3d, Stockholm 1930, Proceedings, v. 1, p. 85-92.

Volman, M. G., 1954, The natural stream of Brandywine Creek, Pennsylvania: U.S. Geological Survey Professional Paper 271, 56 p. 
TABLE 1
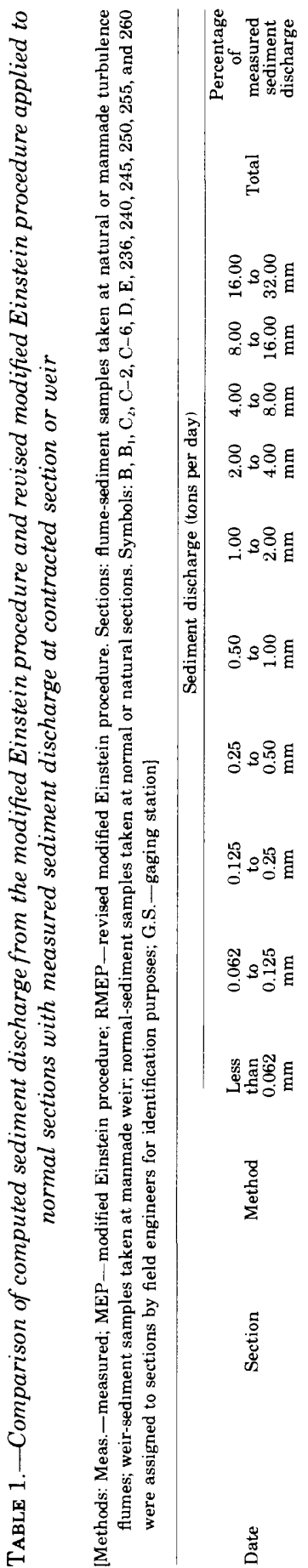

昌告

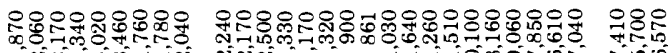

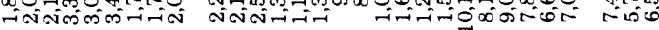

000000000000000000000000000000

000000000000000000000000000000

000000000000000000000000000000

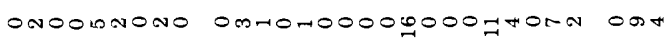

Bூ

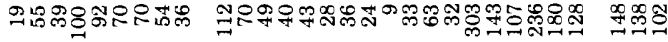

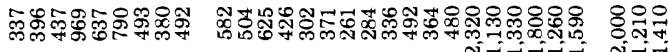

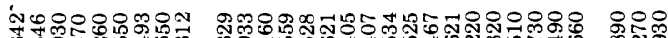

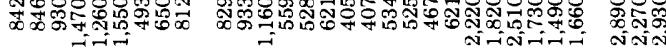

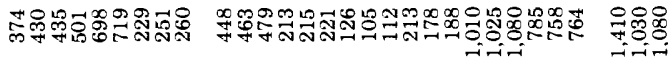

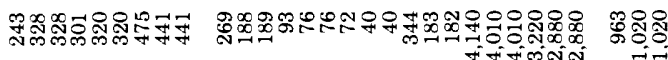

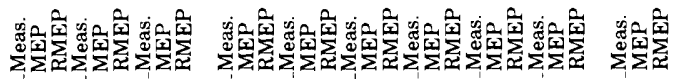

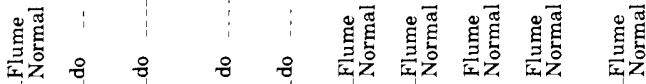

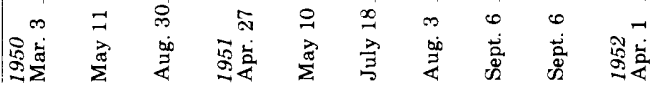




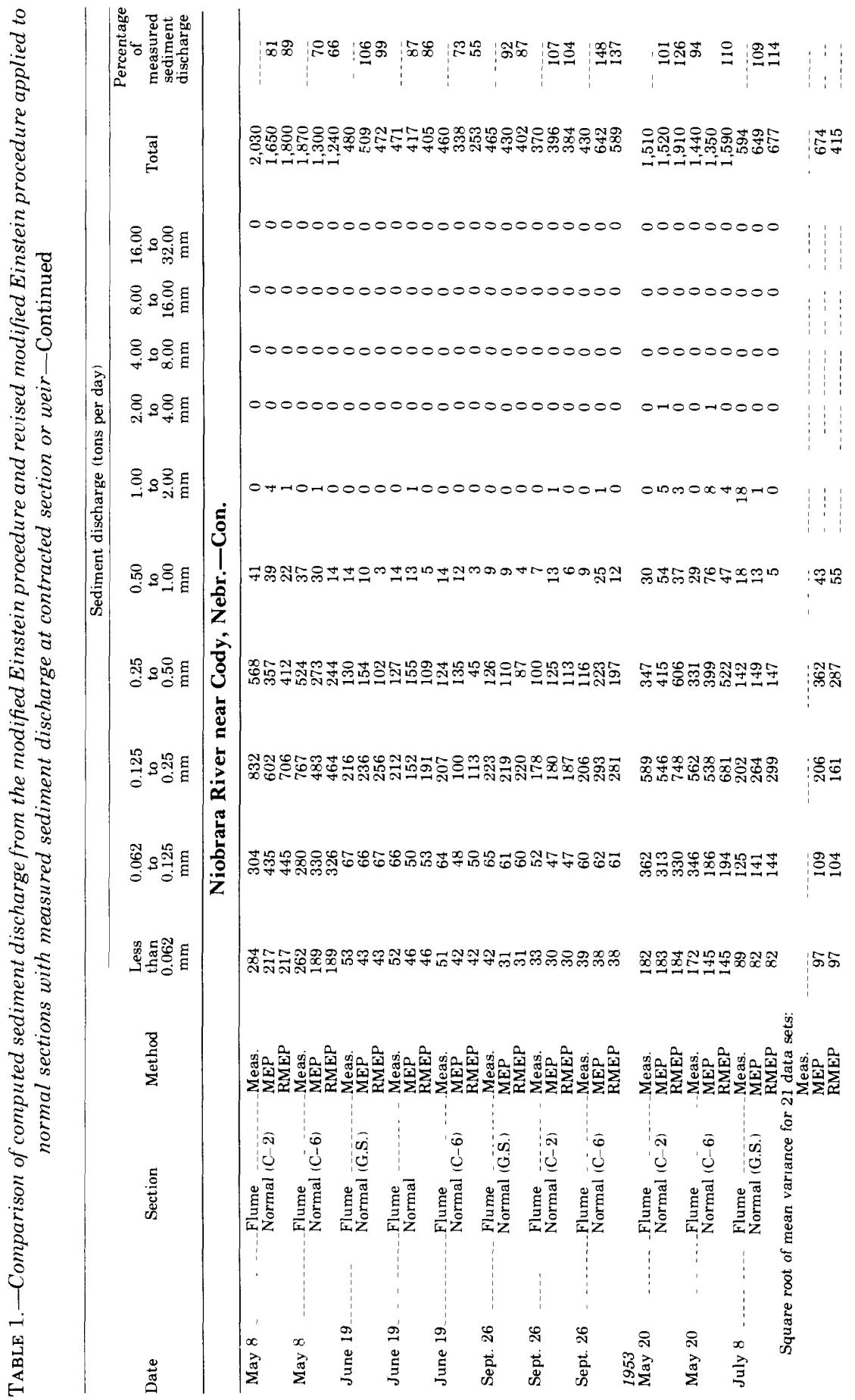




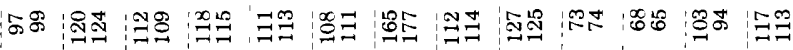

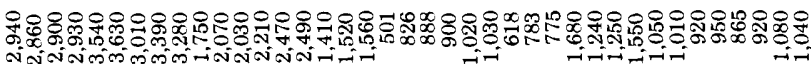

000000000000000000000000000000000000000

000000000000000000000000000000000000000

000000000000000000000000000000000000000

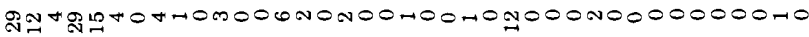

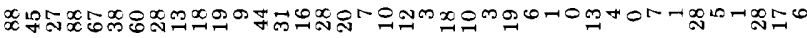

온

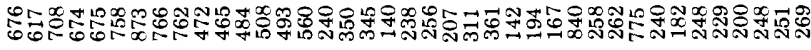

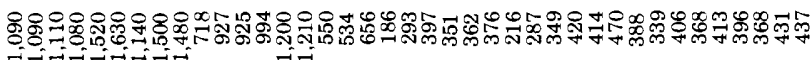

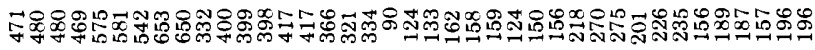

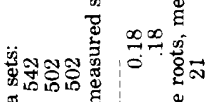

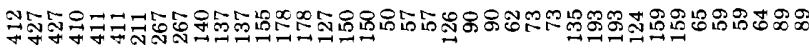

吾

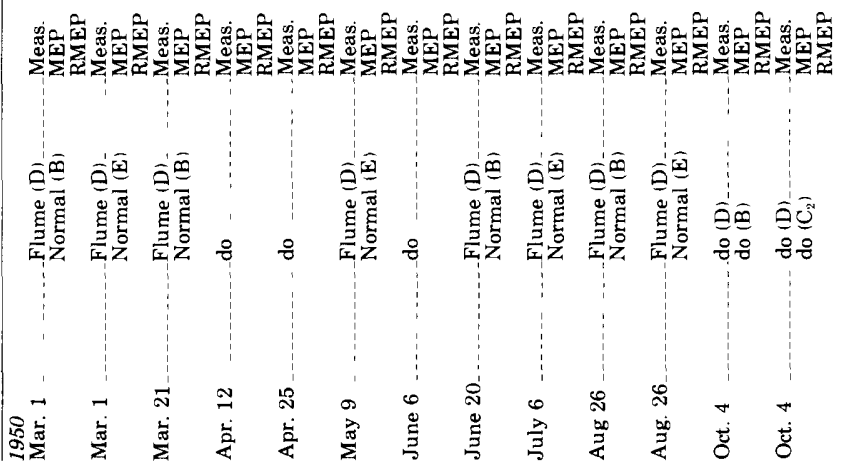


舟

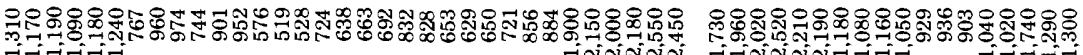

\footnotetext{
000000000000000000000000000000000000000000000000000
}

000000000000000000000000000000000000000000000000000

000000000000000000000000000000000000000000000000000

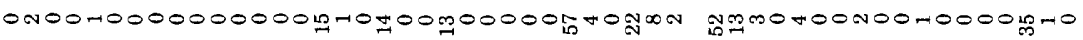

듬

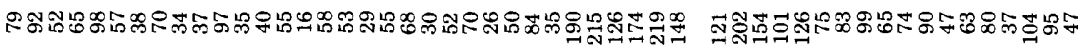

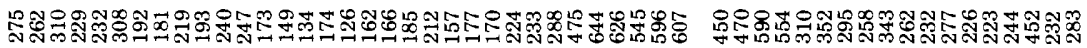

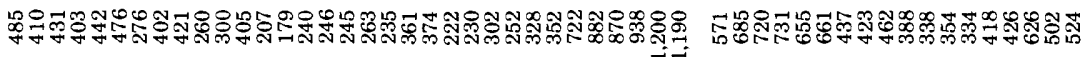

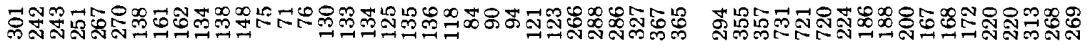

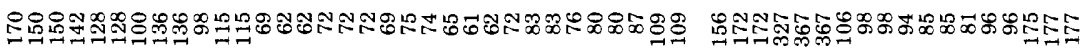

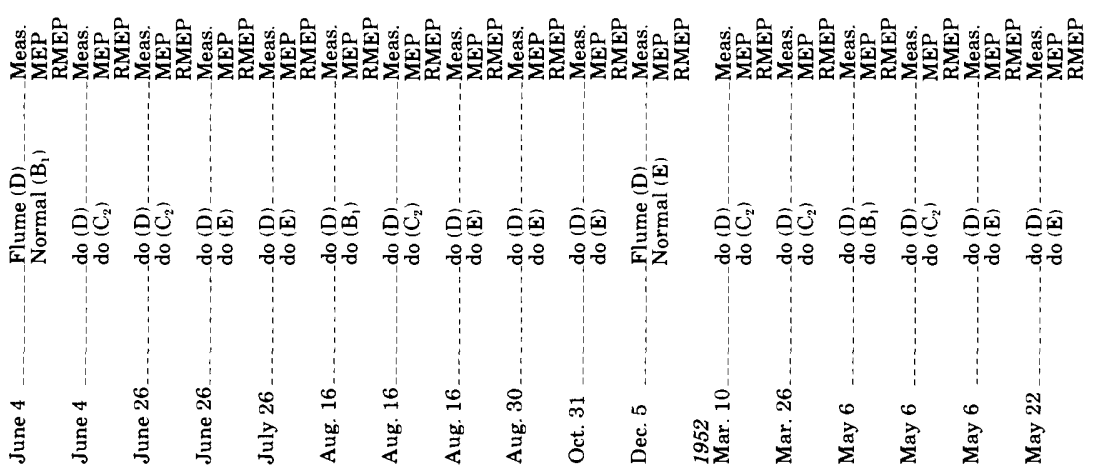




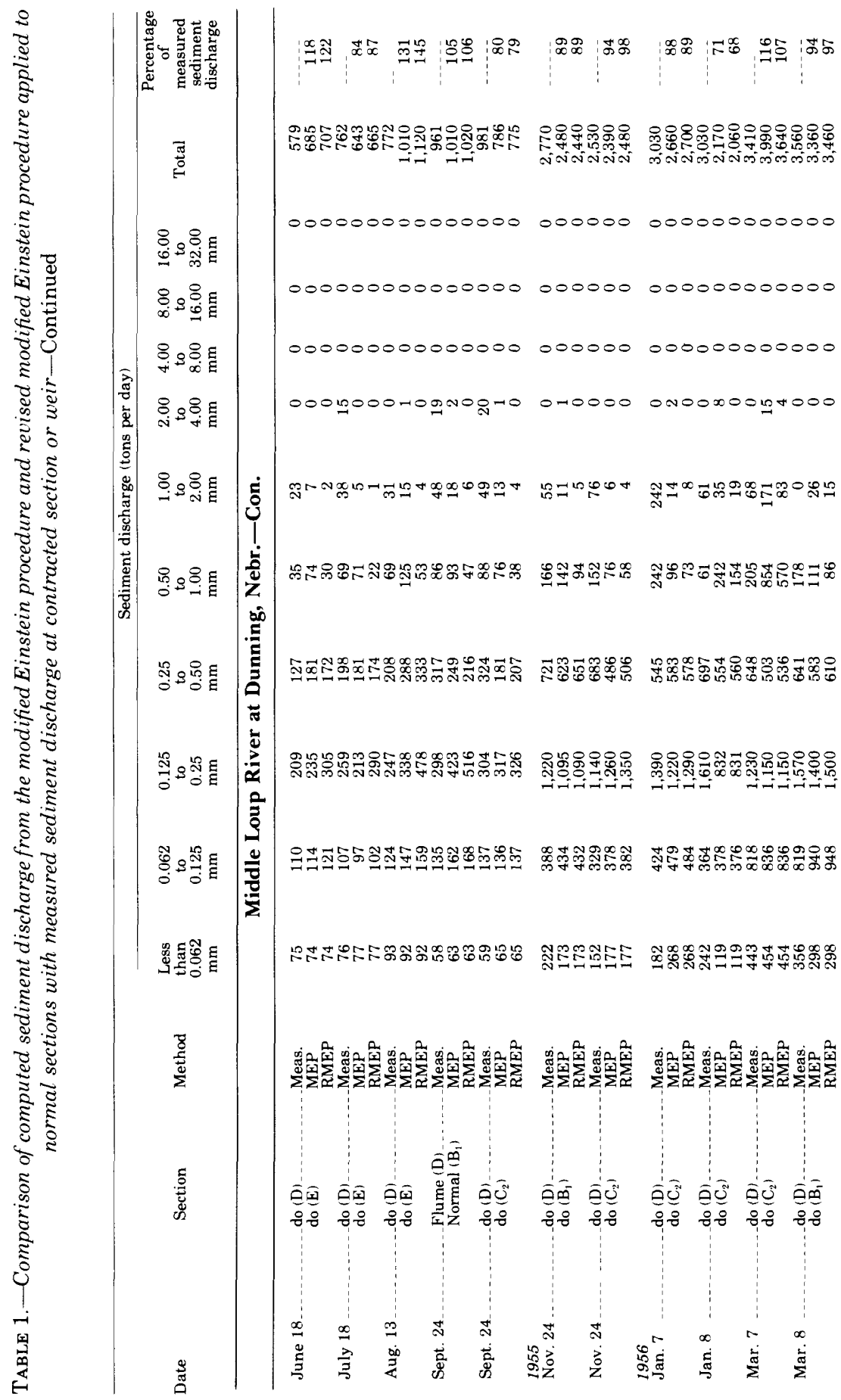


స్తి

:을

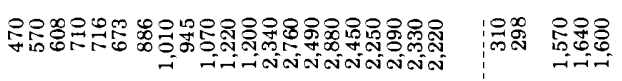

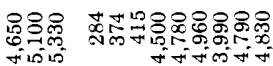

000000000000000000000

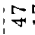

000000000

표 $0000000 \mathrm{No}$

T: 0000 mooman

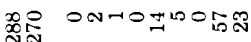

$0000000000000 n 0040$ h40

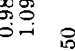

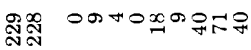

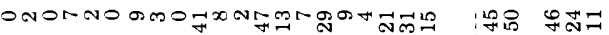

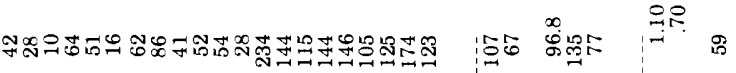

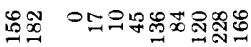

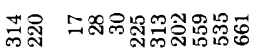

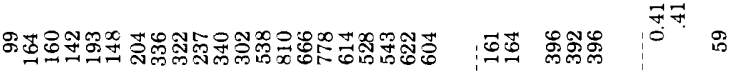

ఈำ.

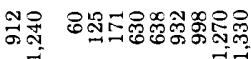
00

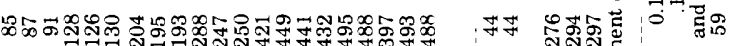

:

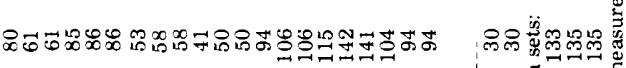

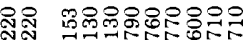
สูก han

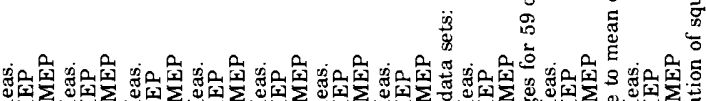




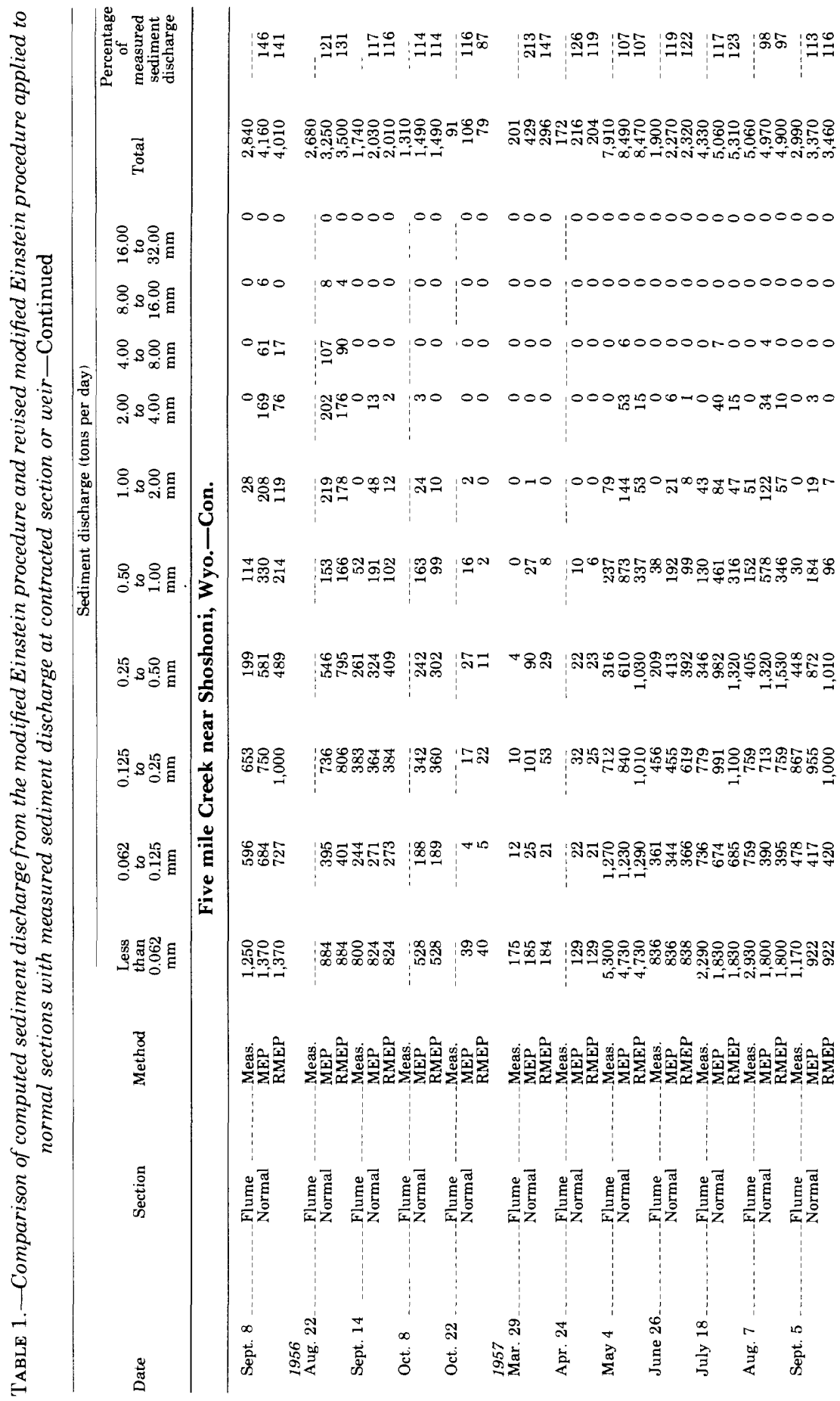




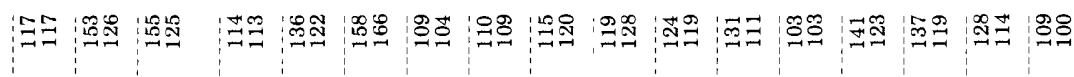

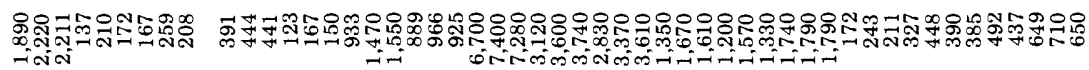

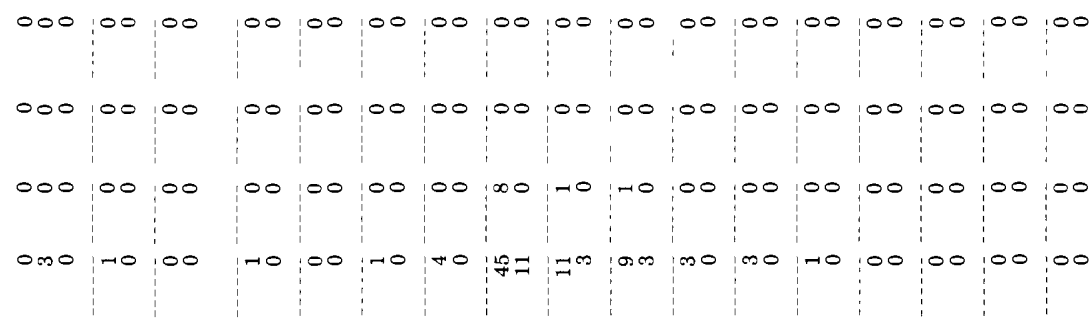

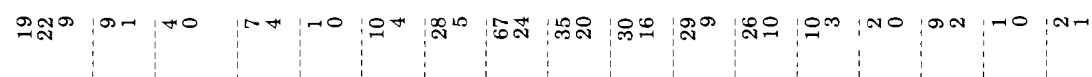

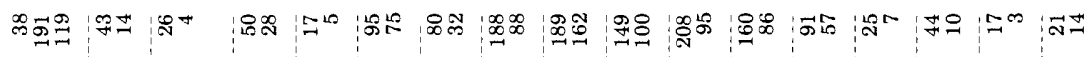

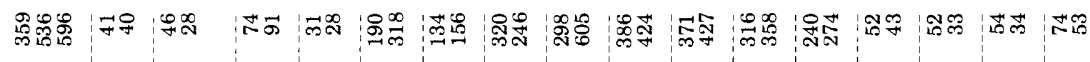

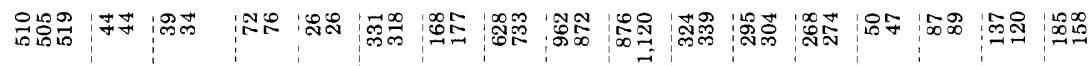

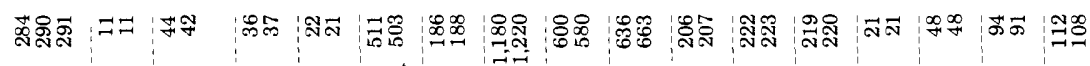
ฉำ

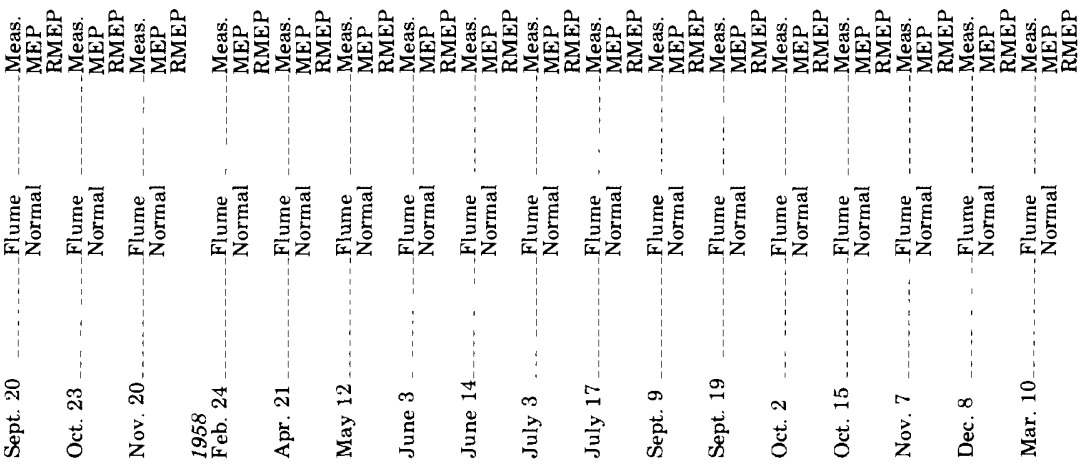




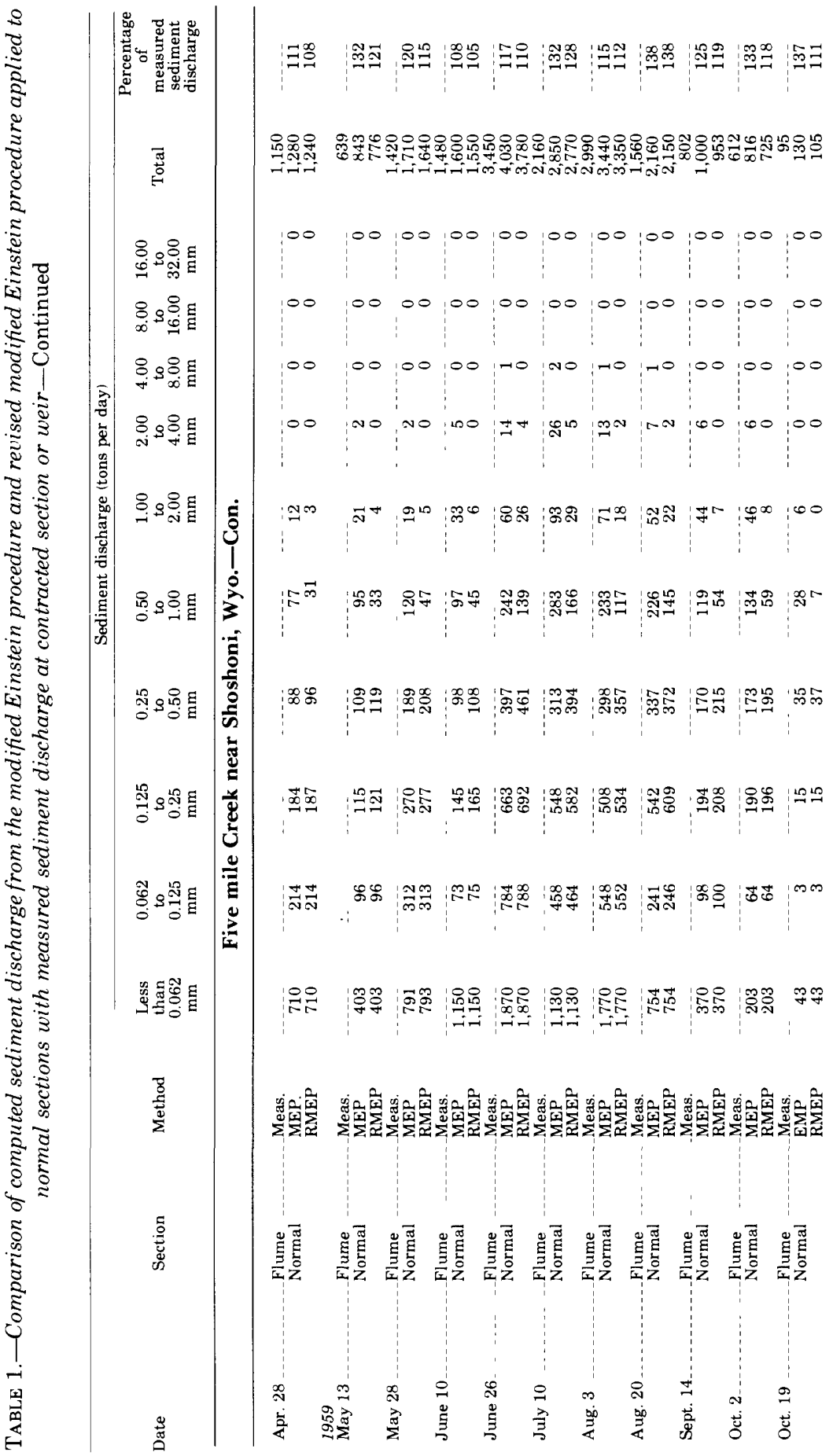




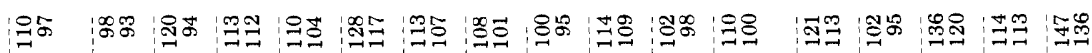

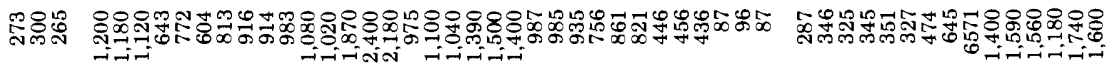

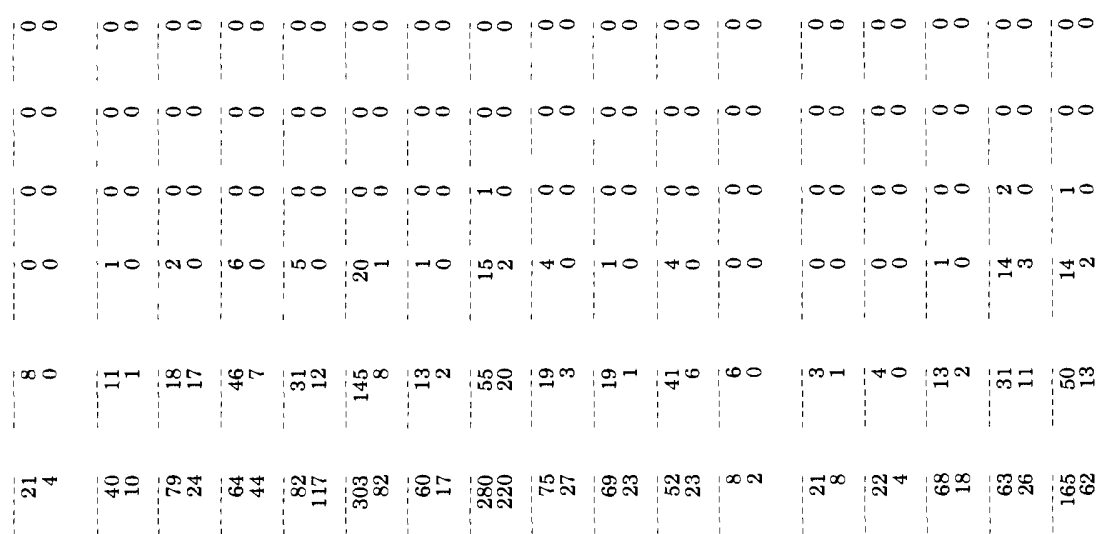

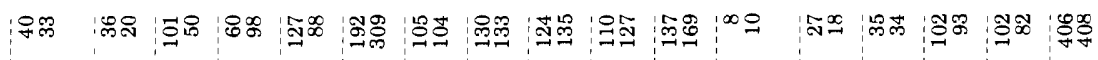

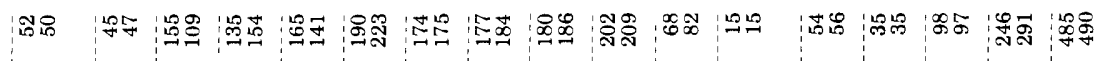

以

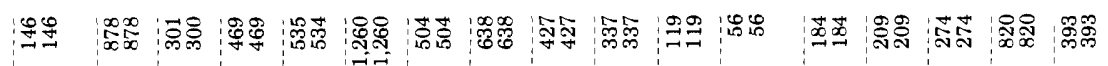

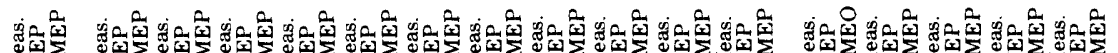

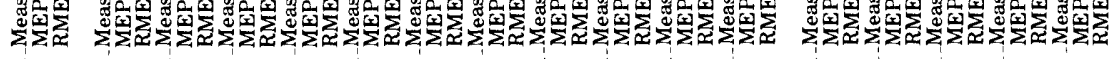

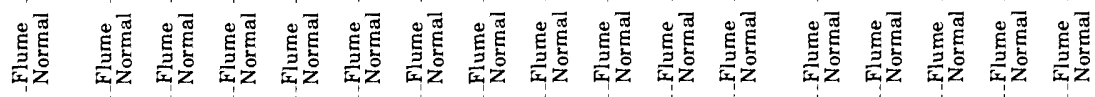

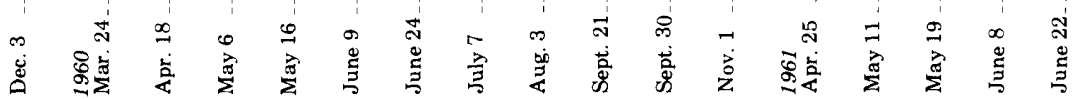




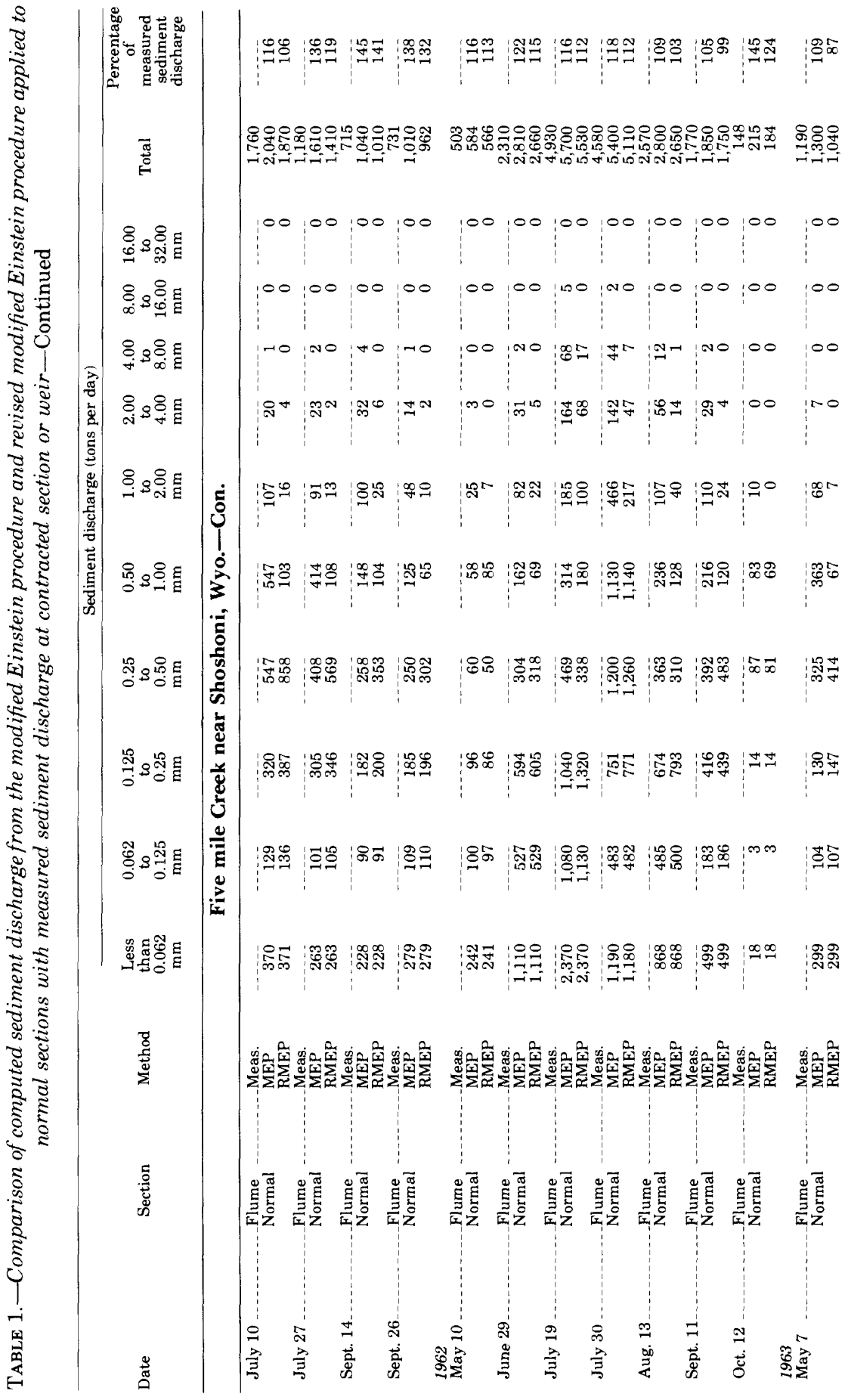


敬

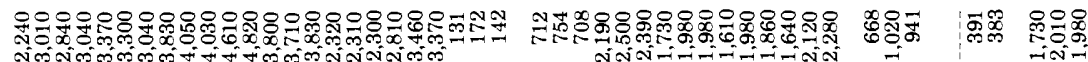

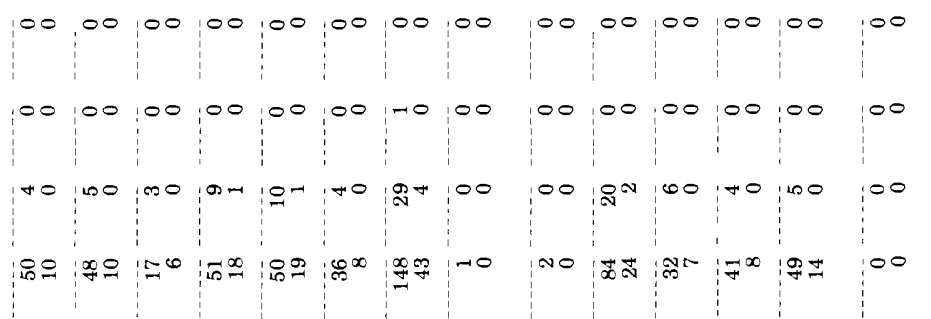

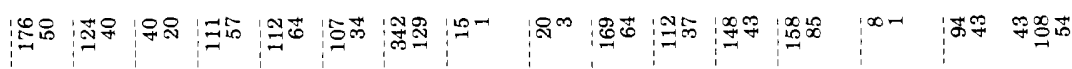

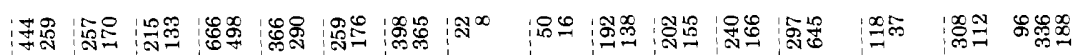

冓 | 壳志 芯

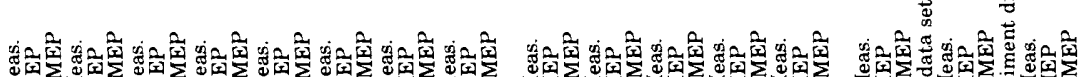

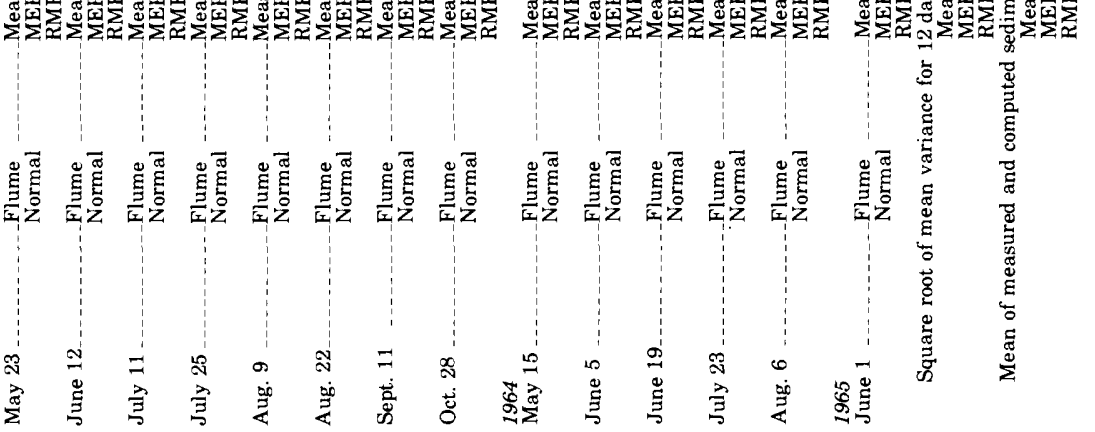




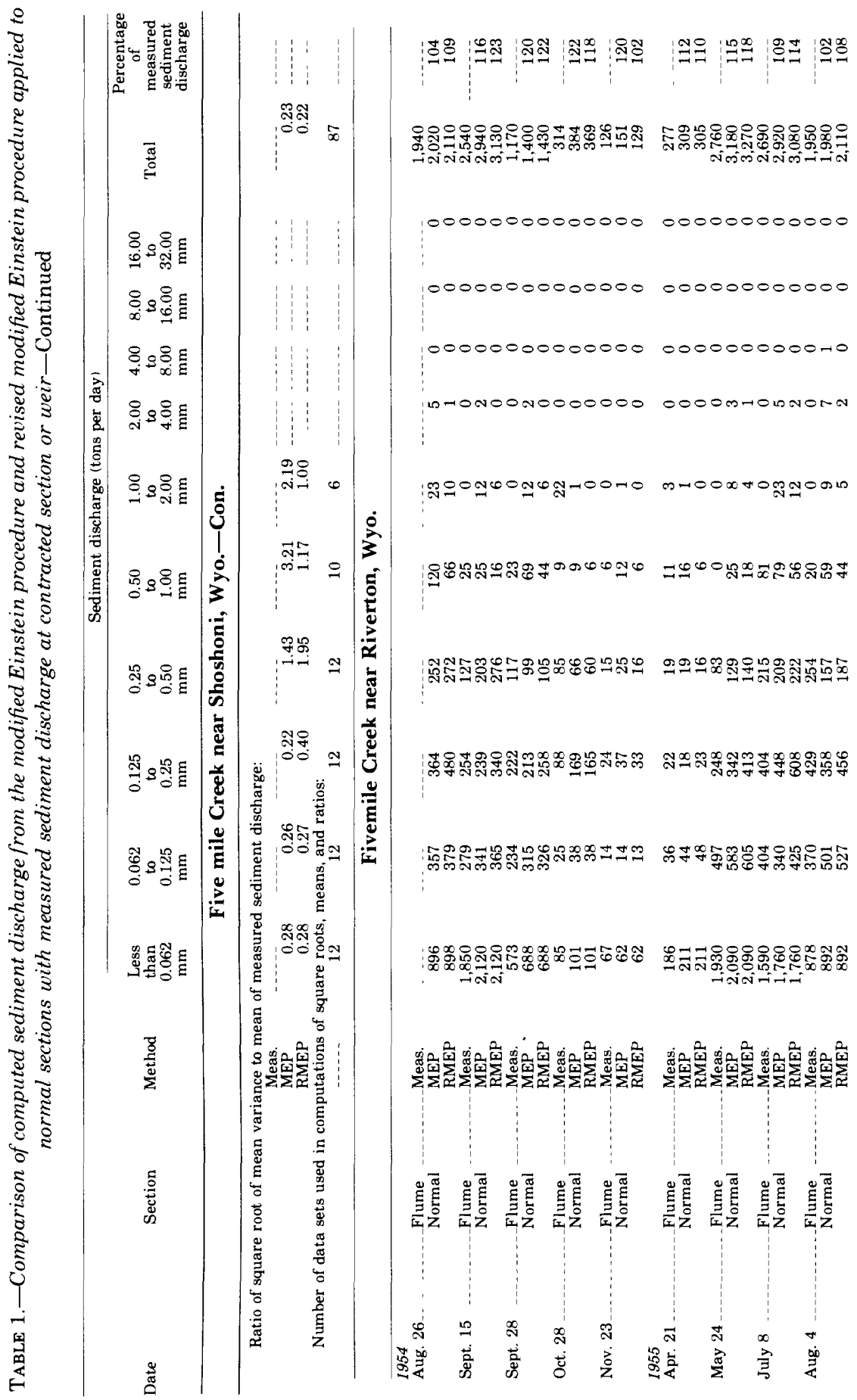




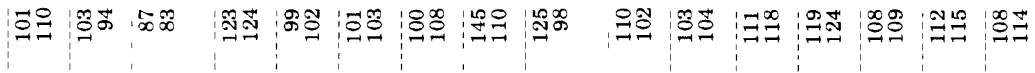

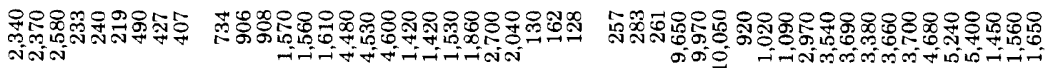

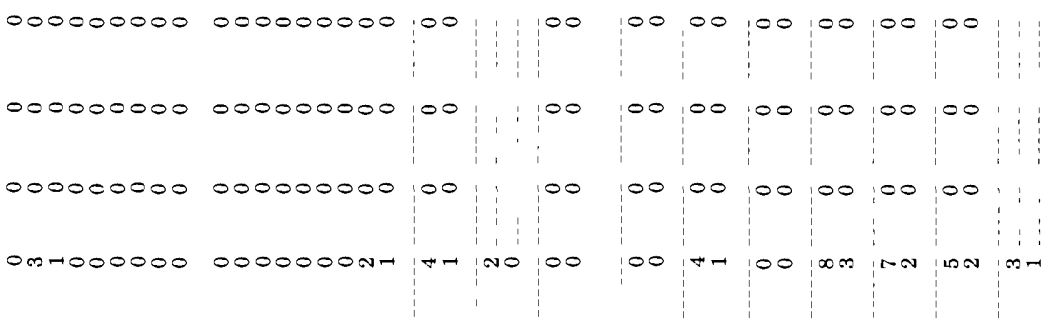

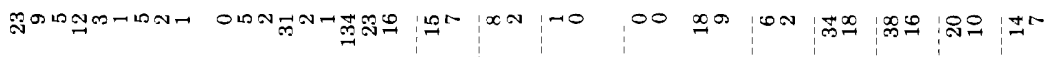

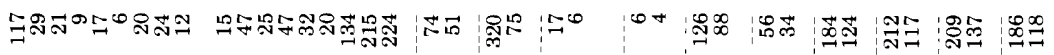

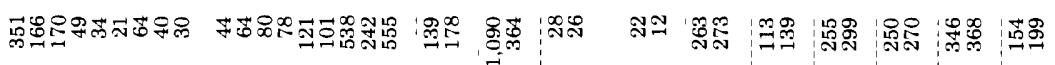

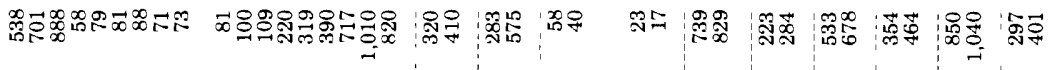

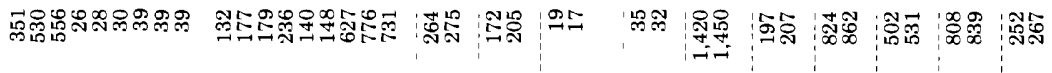

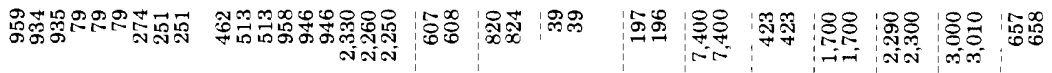

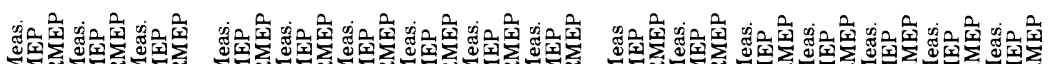

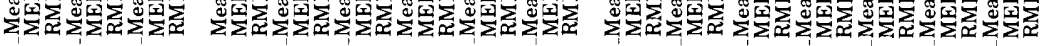

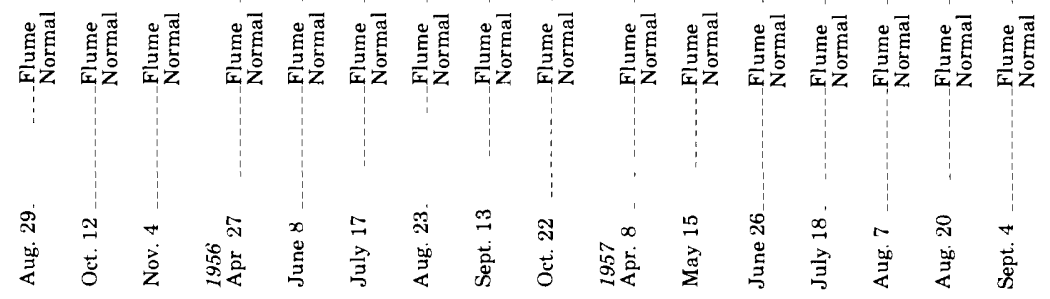




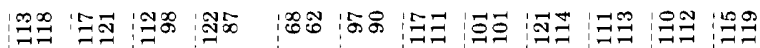

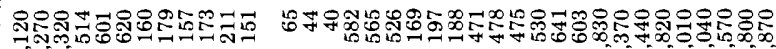

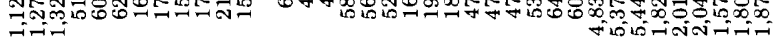

100100100100100100100100100,00100100

$100,00,00,00,00,00,00,00,00,00,00,00$

$100,00: 00,00,100,100,00,00,00,00,00100$

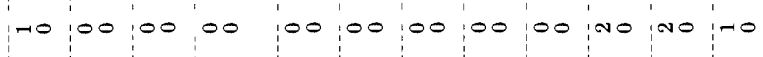

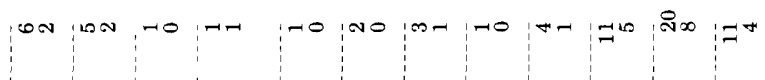

电

谣

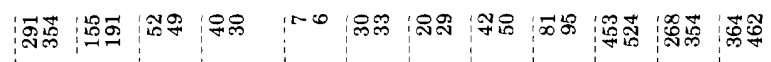

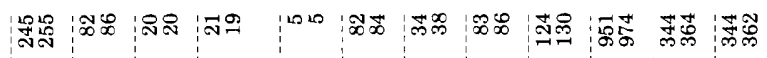

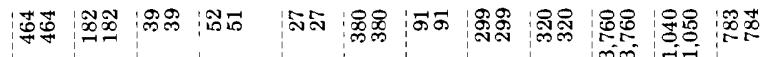

wo

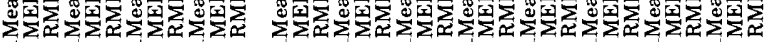

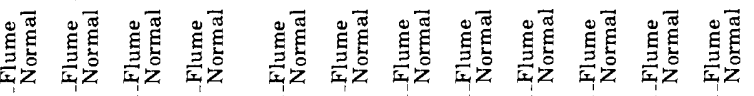

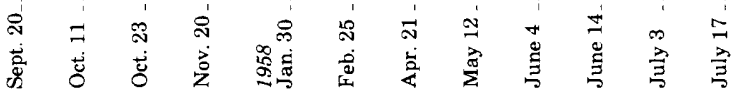


胥

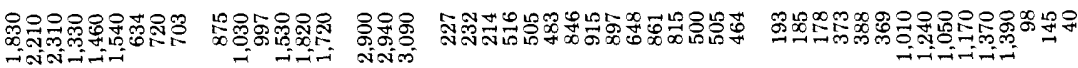

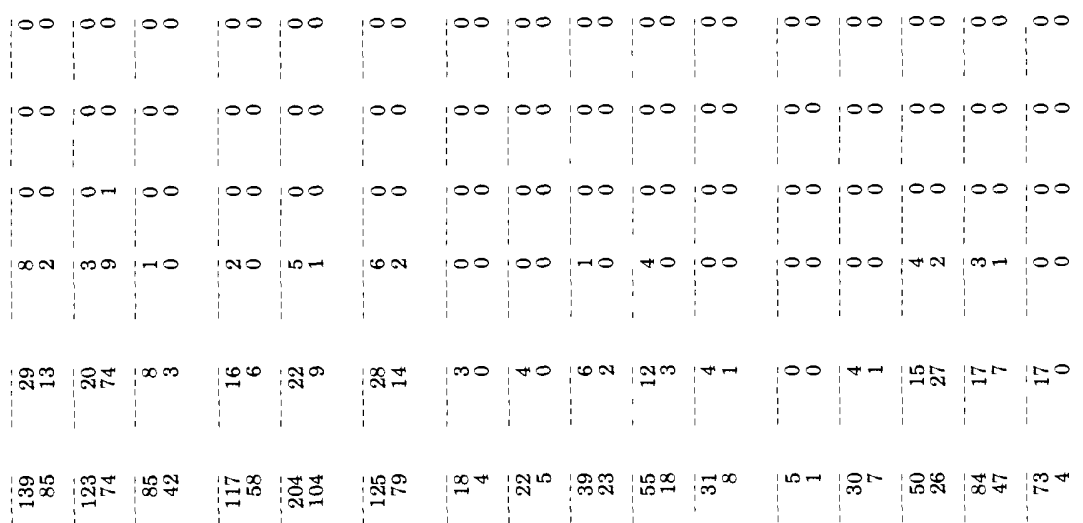


$\stackrel{2}{2}$

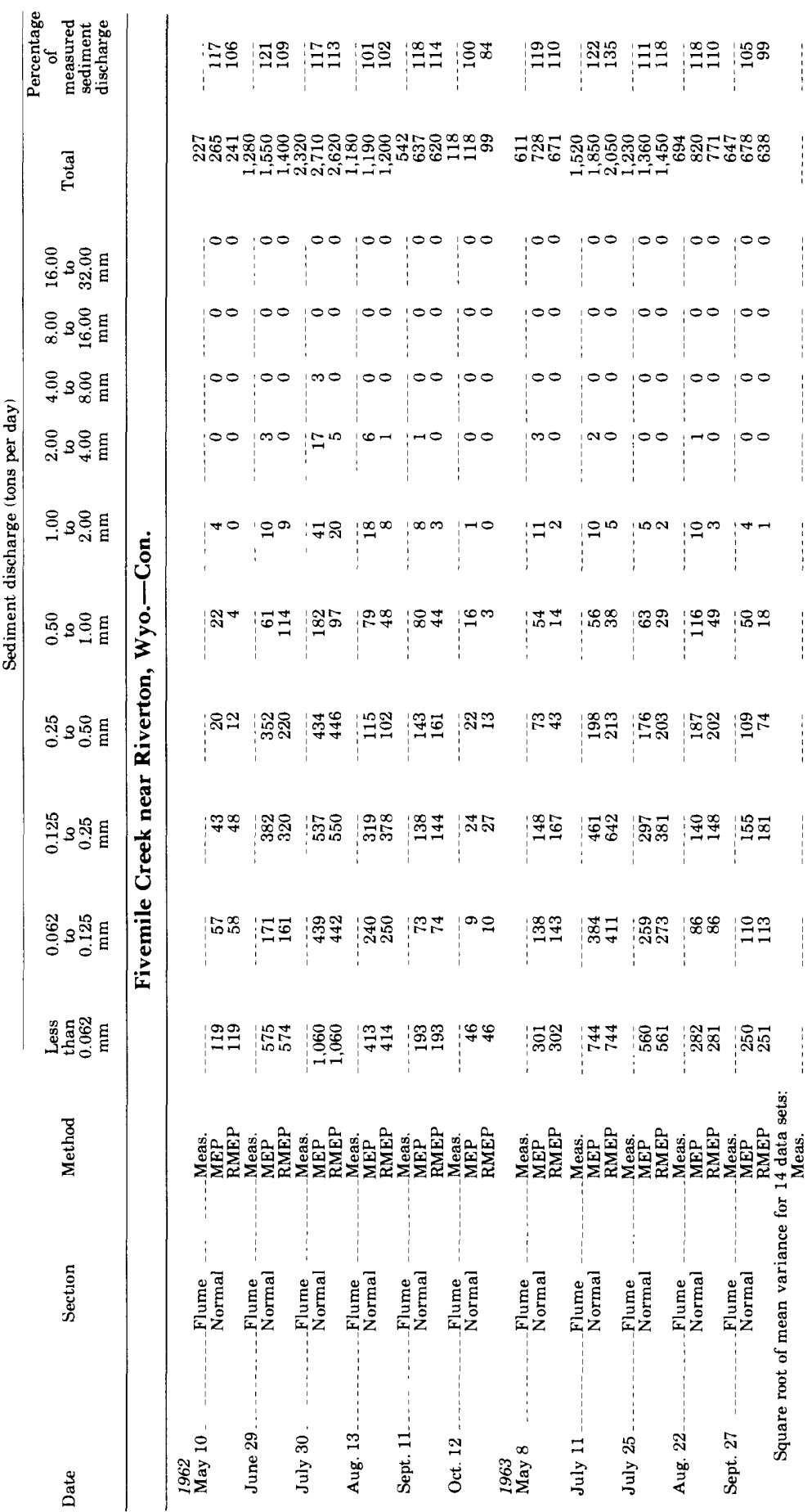




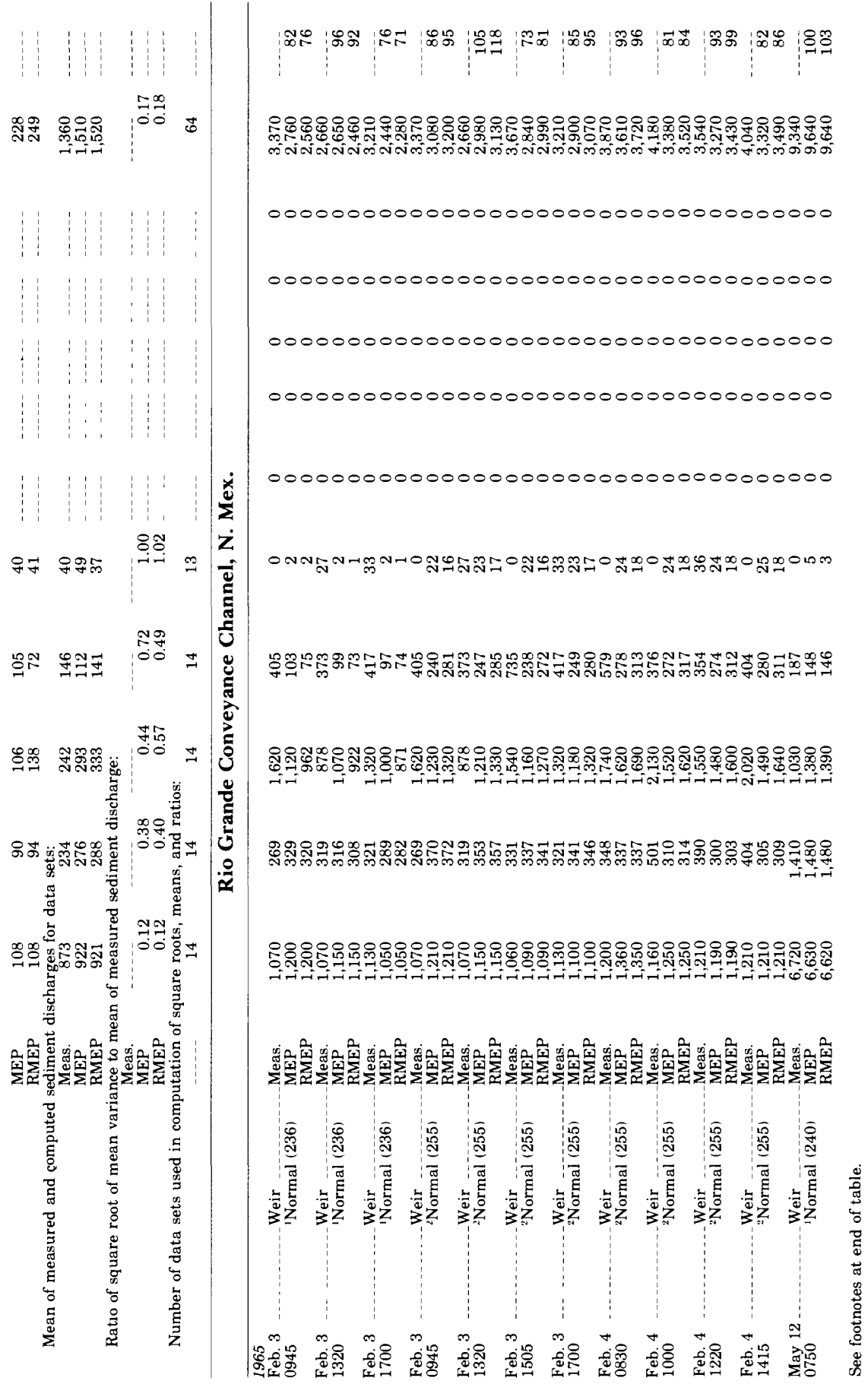


舒

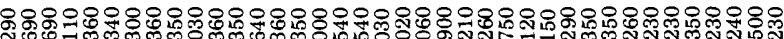

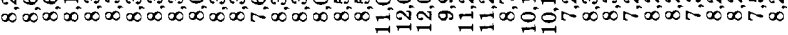

00000000000000000000000000000000000000

00000000000000000000000000000000000000

00000000000000000000000000000000000000

00000000000000000000000000000000000000

0000000000000000000000000000000000000

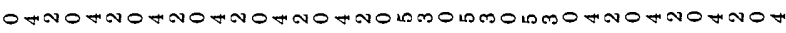

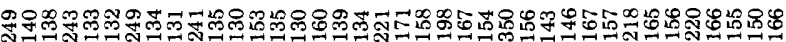

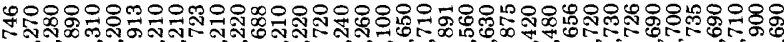

(1)

우유.

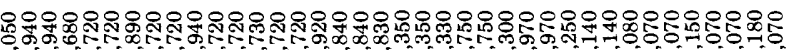

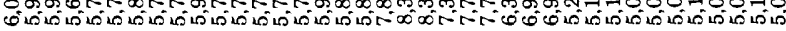

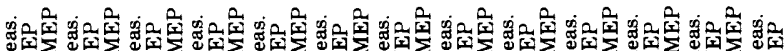

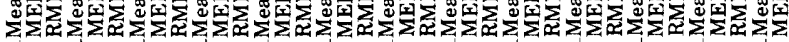


○潘:

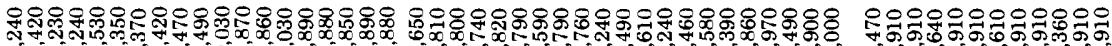

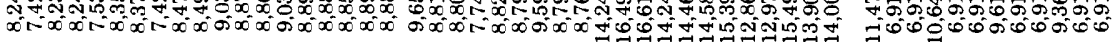

0000000000000000000000000000000000000000000000000000

0000000000000000000000000000000000000000000000000000

0000000000000000000000000000000000000000000000000000

0000000000000000000000000000000000000000000000000000

0000000000000000000000000000000000000000000000000000

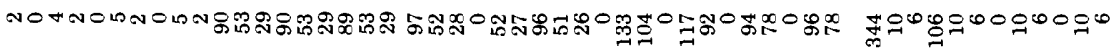

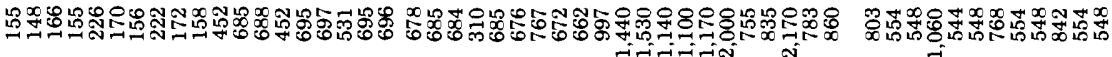

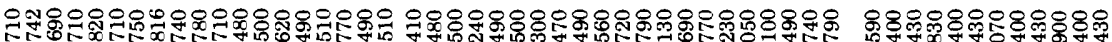
A-

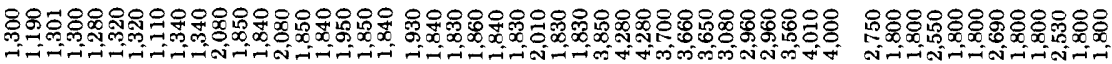

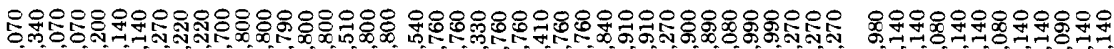

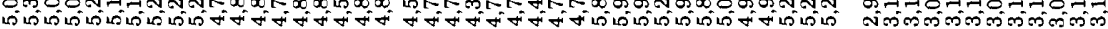

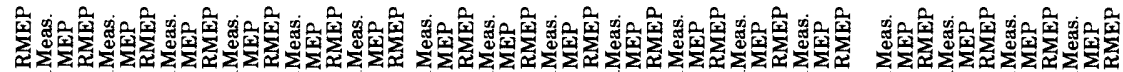

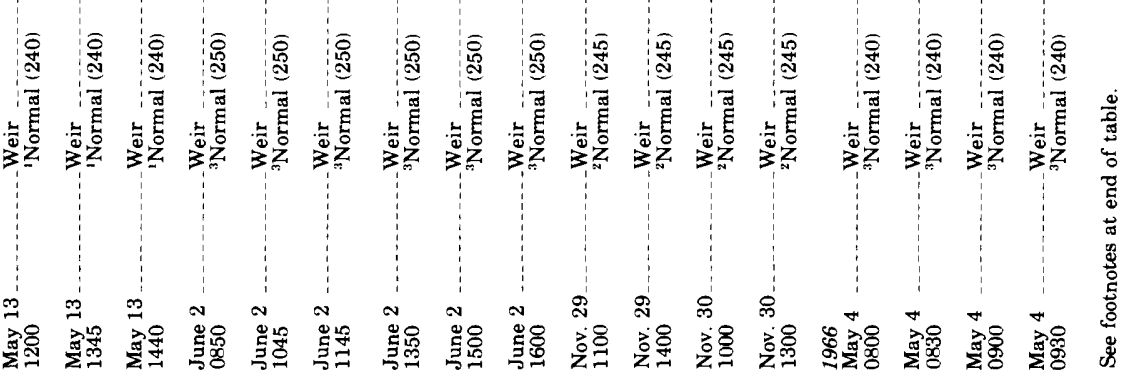




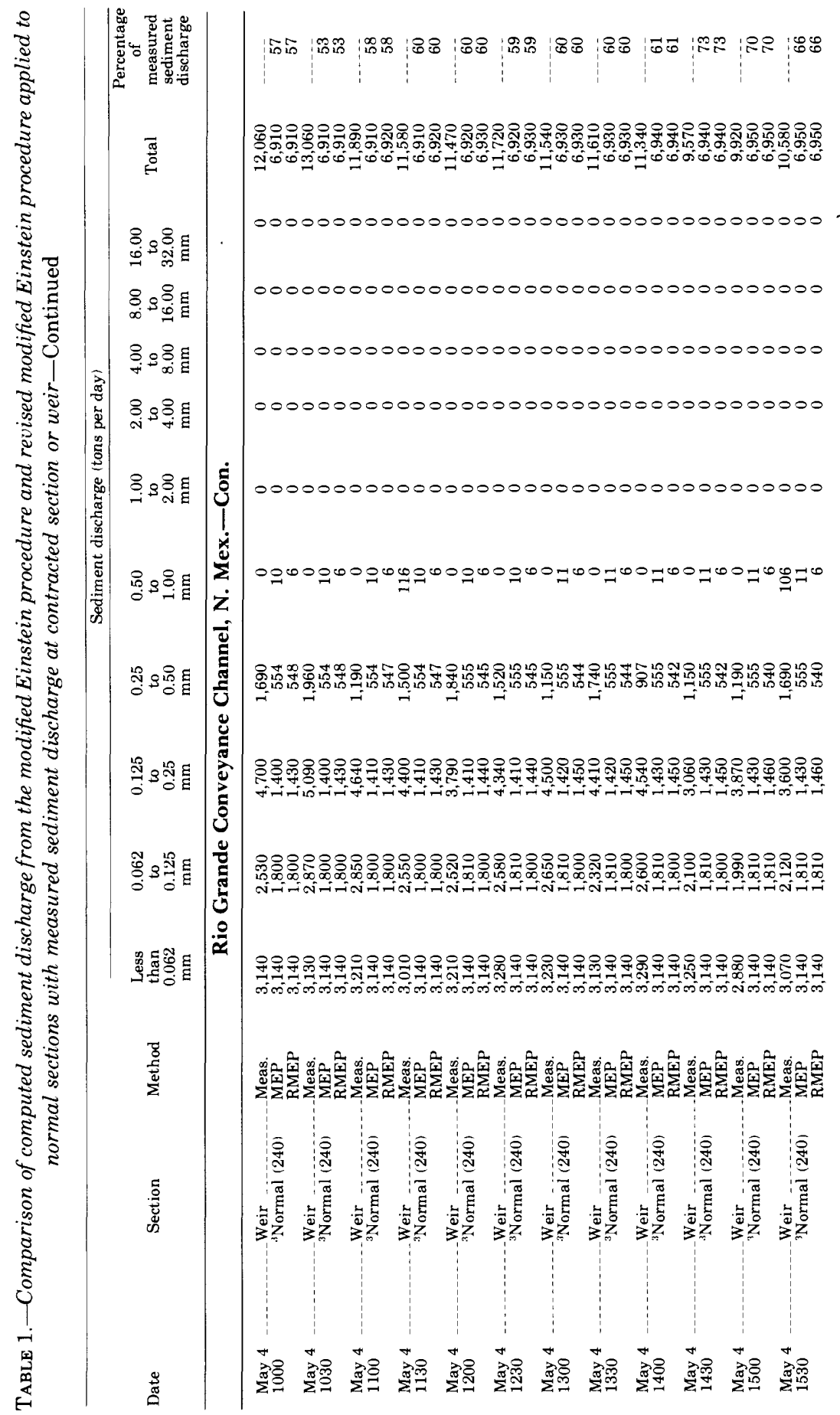




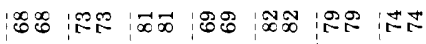

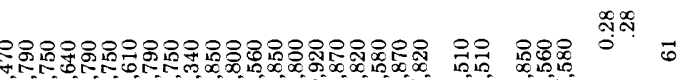

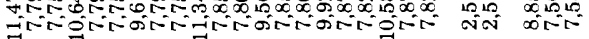

0000000000000000000000000000

0000000000000000000000000000

0000000000000000000000000000

0000000000000000000000000000

0000000000000000000000000000

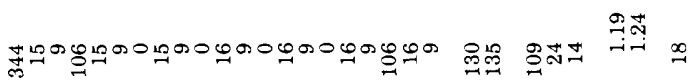

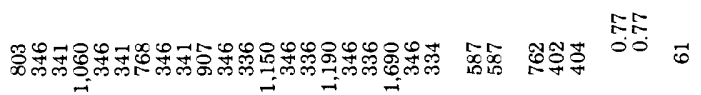

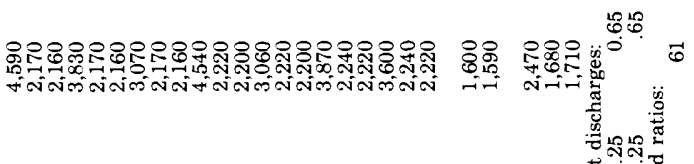

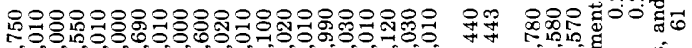

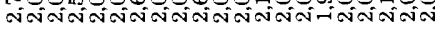

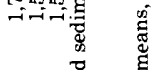
눈용 10

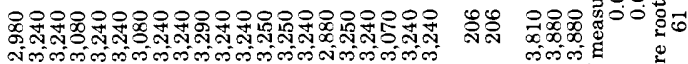
蓆 究

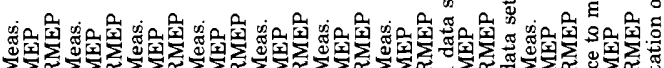

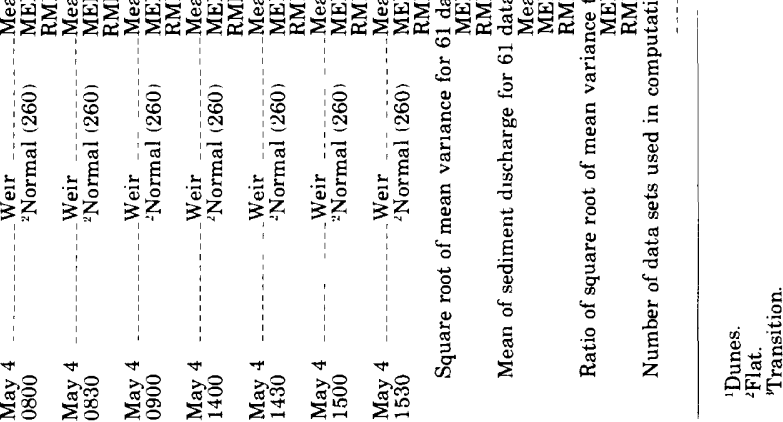

